Western University

Scholarship@Western

Physiology and Pharmacology Publications

Physiology and Pharmacology Department

6-1-2019

\title{
Poly(ester amide) particles for controlled delivery of celecoxib
}

lan J. Villamagna

The University of Western Ontario

Trent N. Gordon

The University of Western Ontario

Mark B. Hurtig

Ontario Veterinary College

Frank Beier

The University of Western Ontario

Elizabeth R. Gillies

The University of Western Ontario

Follow this and additional works at: https://ir.lib.uwo.ca/physpharmpub

Part of the Medical Physiology Commons, and the Pharmacy and Pharmaceutical Sciences Commons

Citation of this paper:

Villamagna, IJ, Gordon, TN, Hurtig, MB, Beier, F, Gillies, ER. 2019. Poly(ester amide) particles for controlled delivery of celecoxib. J Biomed Mater Res Part A 2019: 107A: 1235- 1243. 


\section{Targeting cartilage EGFR pathway for osteoarthritis treatment}

\author{
Yulong Wei ${ }^{1,2,3}$, Lijun Luo ${ }^{2}$, Tao Gui ${ }^{1}$, Feifan Yu ${ }^{4}$, Lesan Yan ${ }^{2}$, Lutian Yao ${ }^{1}$, Leilei Zhong ${ }^{1}$, \\ Wei Yu ${ }^{1,3}$, Biao Han ${ }^{5}$, Jay M. Patel ${ }^{1,6}$, Jessica F. Liu' ${ }^{2}$, Frank Beier ${ }^{7}$, Lawrence Scott Levin ${ }^{1}$, \\ Charles Nelson ${ }^{1}$, Zengwu Shao ${ }^{3}$, Lin Han ${ }^{5}$, Robert L. Mauck ${ }^{1,2,6}$, Andrew Tsourkas ${ }^{2}$, \\ Jaimo Ahn ${ }^{1,8}$, Zhiliang Cheng ${ }^{2 *}$, Ling Qin ${ }^{1 *}$
}

\begin{abstract}
Osteoarthritis (OA) is a widespread joint disease for which there are no disease-modifying treatments. Previously, we found that mice with cartilage-specific epidermal growth factor receptor (EGFR) deficiency developed accelerated knee OA. To test whether the EGFR pathway can be targeted as a potential OA therapy, we constructed two cartilage-specific EGFR overactivation models in mice by overexpressing heparin binding EGF-like growth factor (HBEGF), an EGFR ligand. Compared to wild type, Col2-Cre HBEGF-overexpressing mice had persistently enlarged articular cartilage from adolescence, due to an expanded pool of chondroprogenitors with elevated proliferation ability, survival rate, and lubricant production. Adult Col2-Cre HBEGF-overexpressing mice and Aggrecan-CreER HBEGF-overexpressing mice were resistant to cartilage degeneration and other signs of $O A$ after surgical destabilization of the medial meniscus (DMM). Treating mice with gefitinib, an EGFR inhibitor, abolished the protective action against OA in HBEGF-overexpressing mice. Polymeric micellar nanoparticles (NPs) conjugated with transforming growth factor- $\alpha$ (TGF $\alpha$ ), a potent EGFR ligand, were stable and nontoxic and had long joint retention, high cartilage uptake, and penetration capabilities. Intra-articular delivery of TGF $\alpha$-NPs effectively attenuated surgery-induced OA cartilage degeneration, subchondral bone plate sclerosis, and joint pain. Genetic or pharmacologic activation of EGFR revealed no obvious side effects in knee joints and major vital organs in mice. Together, our studies demonstrate the feasibility of using nanotechnology to target EGFR signaling for OA treatment.
\end{abstract}

\section{INTRODUCTION}

Osteoarthritis $(\mathrm{OA})$ is the most common chronic condition of the joints, affecting 303 million people globally in 2017 (1). As a joint degenerative disease, it is primarily characterized by destruction of articular cartilage but is often accompanied by subchondral bone thickening, osteophyte formation, synovial inflammation, and hypertrophy of the joint capsule (2). Posttraumatic OA develops after joint injury and causes an acute form of cartilage degeneration. Patients with OA experience pain and disability, for which there are neither cures nor disease-modifying treatments.

The outermost superficial layer of articular cartilage is the first line of defense against OA initiation. Distinct from other layers of articular cartilage (transitional, middle, and calcified zones), this twoto four-cell-thick superficial zone has distinct biologic and mechanical functions, such as producing lubricant proteins, harboring chondroprogenitors, resisting shear stresses, and serving as a gliding surface (3). In OA, degenerative changes are initiated with cellular disorganization and irregular surface of the superficial layer (4-6).

The epidermal growth factor receptor (EGFR) is one of the most well-studied signaling pathways. Upon activation by its ligands,

\footnotetext{
${ }^{1}$ Department of Orthopaedic Surgery, Perelman School of Medicine, University of Pennsylvania, Philadelphia, PA 19104, USA. ${ }^{2}$ Department of Bioengineering, School of Engineering and Applied Science, University of Pennsylvania, Philadelphia, PA 19104, USA. ${ }^{3}$ Department of Orthopaedics, Union Hospital, Tongji Medical College, Huazhong University of Science and Technology, Wuhan 430022, China. ${ }^{4}$ Alphathera LLC, Philadelphia, PA 19146, USA. ${ }^{5}$ School of Biomedical Engineering, Science and Health Systems, Drexel University, Philadelphia, PA 19104, USA. ${ }^{6}$ Translational Musculoskeletal Research Center, Corporal Michael J. Crescenz VA Medical Center, Philadelphia, PA 19104, USA. ${ }^{7}$ Department of Physiology and Pharmacology, Schulich School of Medicine and Dentistry, University of Western Ontario, London, Ontario N6A 5C1, Canada. ${ }^{8}$ Department of Orthopaedic Surgery, University of Michigan, Ann Arbor, MI 48104, USA. *Corresponding author. Email: qinling@pennmedicine.upenn.edu (L.Q.); zcheng@seas. upenn.edu (Z.C.)
}

EGFR dimerizes with itself or other members of the EGFR family to modulate a variety of cellular functions, such as proliferation, survival, adhesion, migration, and differentiation (7). Mice lacking EGFR activity specifically in cartilage (Col2-Cre) have fewer superficial chondrocytes, less secretion of boundary lubrication, and weak mechanical strength at the cartilage surface than their wildtype (WT) siblings (8). With a defective articular cartilage surface, these mice develop markedly accelerated knee OA during aging or after OA injury $(8,9)$. Mig6 is a negative feedback inhibitor of EGFR (10). Consistently, mice with Mig6 deficiency in cartilage or limb bud mesenchyme display thickened articular cartilage with a drastically increased number of superficial chondrocytes at a young age (11-13). Together, these lines of evidence suggest that the EGFR pathway is critical for maintaining homeostasis of the superficial layer of articular cartilage.

We hypothesized that targeting the EGFR pathway could be an effective OA therapy. To test this, we designed a two-pronged approach. First, we genetically enhanced EGFR activity by adopting a Rosa-diphtheria toxin receptor $(D T R)$ model that is normally used for cell ablation (14). Originally identified as a receptor for bacterial diphtheria toxin, DTR was later found to be human full-length heparin binding EGF-like growth factor (HBEGF) (15), a ligand for EGFR. Human HBEGF shares $81 \%$ sequence identity with the mouse ortholog. Breeding Rosa-DTR mice with cartilage-specific Col2-Cre or Aggrecan-CreER mice allowed us to study the effect of cartilage-specific EGFR overactivation on OA progression. Second, we developed an advanced nanoparticle (NP)-based system for the local delivery of an EGFR ligand, transforming growth factor- $\alpha$ (TGF $\alpha$ ), into knee cartilage. Because TGF $\alpha$, the most highly expressed EGFR ligand in mouse cartilage (8), has a short life span of $20 \mathrm{~min}$ in the circulation (16), it is not feasible to use TGF $\alpha$ directly 
as an OA drug. Recently, NP-based targeted drug delivery has been pursued in the treatment of OA (17-19). A strength of these delivery systems is the prolonged retention of drugs in the joints, thus lowering the overall therapeutic dose required and reducing off-target toxicity and side effects. To increase the retention and minimize the leakage of TGF $\alpha$ from the joint space, we synthesized TGF $\alpha$-NPs by conjugating TGF $\alpha$ onto the surfaces of polymeric micellar NPs. We then characterized the properties of these TGF $\alpha$-NPs in the joint tissue and performed a proof-of-principle study by testing their efficacy in attenuating OA progression in a mouse injury model.

\section{RESULTS}

\section{Overexpression of HBEGF in chondrocytes leads to cartilage enlargement}

To target the EGFR pathway in cartilage, we generated Col2-Cre Rosa-DTR mice [Col2-Cre Rosa-HBEGF (HBEGF Over ${ }^{\text {Col2 }}$ ) mice].
These mice had similar body weight and body length as WT mice (fig. S1). Western blots (Fig. 1A) and immunohistochemistry (IHC; fig. S2) confirmed that there was increased HBEGF in chondrocytes, leading to EGFR activation as shown by elevated phosphoEGFR (p-EGFR) and phospho-extracellular signal-regulated kinase (p-ERK) amounts (Fig. 1A). HBEGF Over ${ }^{\mathrm{Col} 2}$ mice at 5 and 12 months of age displayed normal knee joints (Fig. 1B) without gross abnormalities, such as osteophytes or synovitis (fig. S3). Long bone structure, including subchondral trabecular bone, subchondral bone plate (SBP), and metaphyseal trabecular bone, was also not affected (fig. S4).

The most obvious change in the HBEGF Over ${ }^{\text {Col2 }}$ skeleton is cartilage. At 1 month of age, its growth plate was modestly expanded by $16 \%$, mainly due to the elongation of the proliferative zone (Fig. 1, C to E). On the contrary, its hypertrophic zone was shrunk. At 5 months of age, the expansion of growth plate was more obvious (2.41-fold). Similar expansion was also observed in articular cartilage (23 and $34 \%$ increases in total cartilage thickness at 1 and
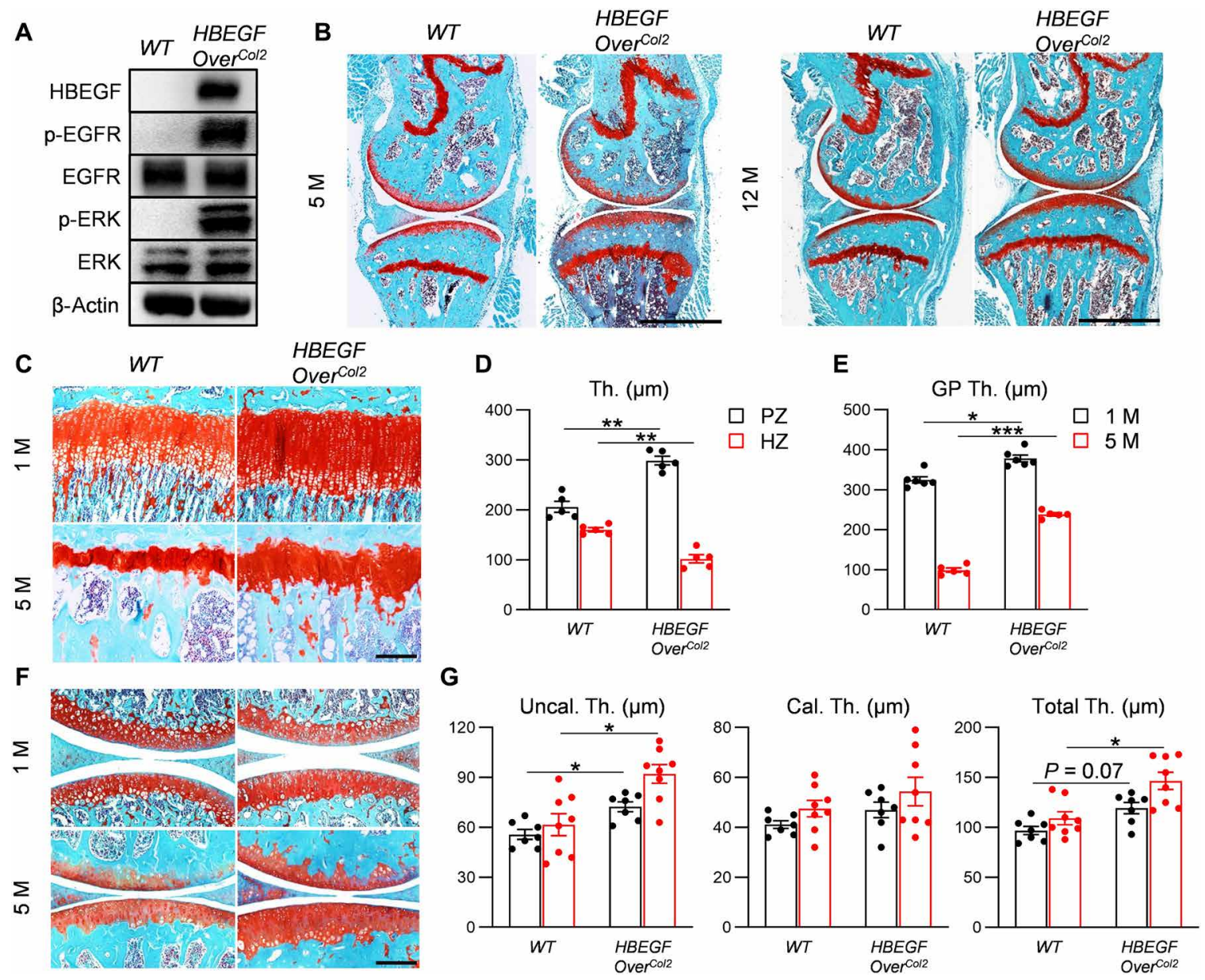

Fig. 1. Overexpression of HBEGF in chondrocytes expands mouse growth plate and articular cartilage without affecting the gross appearance of knee joints. (A) Western blot of HBEGF and EGFR downstream signals (p-EGFR and p-ERK) in articular cartilage chondrocytes derived from 5-month-old $H B E G F$ Over ${ }^{C O / 2}$ mice. $n=3$ independent experiments. (B) Safranin O/Fast Green staining of knee joints from 5- or 12-month-old HBEGF Over ${ }^{\text {Col2 }}$ and control littermates (WT). M, month. Scale bars, $1 \mathrm{~mm} . n=3$ mice per group. (C) Safranin O/Fast Green staining of tibial growth plate in WT and HBEGF Over ${ }^{\text {Col2 }}$ mice at 1 and 5 months of age. Scale bar, $200 \mu \mathrm{m}$. (D) The thicknesses (Th.) of the proliferative zone (PZ) and hypertrophic zone (HZ) in the growth plate of 1-month-old mice. $n=5$ mice per group. (E) The growth plate thickness (GP Th.) quantified from 1- and 5-month-old mice. $n=5$ mice per group. (F) Safranin O/Fast Green staining of articular cartilage in WT and HBEGF Over ${ }^{\text {Col2 }}$ knee joints at 1 and 5 months of age. Scale bar, $200 \mu \mathrm{m}$. (G) Average thicknesses of the uncalcified zone (Uncal. Th.), calcified zone (Cal. Th.), and total tibial articular cartilage quantified from 1 - and 5-month-old mice. $n=8$ mice per group. Statistical analysis was performed using two-way ANOVA with Bonferroni's post hoc analysis. Data are presented as means \pm SEM. ${ }^{*} P<0.05,{ }^{* *} P<0.01$, and ${ }^{* * *} P<0.001$. 
5 months of age, respectively) with a major change in uncalcified cartilage but not in calcified cartilage (Fig. 1, F and G).

\section{EGFR overactivation expands the chondroprogenitor pool}

The superficial layer contains chondroprogenitors responsible for forming cells in the rest of articular cartilage during development (20). Our previous study showed that EGFR inactivation in chondrocytes (Col2-Cre CKO) leads to fewer superficial chondrocytes (8). In $W T$ joints, the number of chondrocytes in the superficial zone declined by $39 \%$ during cartilage maturation (Fig. 2, A and B). This decline did not occur in HBEGF Over ${ }^{\mathrm{Col} 2}$ mice, which exhibited a 1.79-fold increase in superficial chondrocytes compared to WT at 5 months of age (Fig. 2, A and B). This was accompanied by enhanced Ki67 and proteoglycan 4 (PRG4) staining and reduced terminal deoxynucleotidyl transferase deoxyuridine triphosphate nick end labeling (TUNEL) staining (Fig. 2, C and D) in HBEGF Over ${ }^{\mathrm{Col} 2}$ mice, suggesting that constitutive overactivation of EGFR signaling promotes proliferation, survival, and lubricant synthesis in chondrocytes.

5-Ethynyl-2'-deoxyuridine (EdU) labels cells undergoing proliferation. At 1 week of age, $46 \%$ of periarticular cells in epiphyseal cartilage, the site for future articular cartilage, were labeled in WT mice (Fig. 2, E and F). Three weeks later when articular cartilage is established, $27 \%$ of chondrocytes (most of them in uncalcified cartilage) were still labeled by EdU, indicating that many proliferative chondrocytes are slow-cycling cells, i.e., chondroprogenitors. HBEGF Over ${ }^{\mathrm{Col} 2}$ mice had more EdU ${ }^{+}$cells than $W T$ mice at 4 weeks of age (Fig. 2, E and F). After dissection and digestion, 5-month-old HBEGF Over ${ }^{\text {Col2 } 2}$ cartilage formed 1.96-fold more colony-forming unit fibroblast (CFU-F) colonies than WT cartilage in culture (Fig. 2, G and $\mathrm{H}$ ). In addition, progenitors from $\mathrm{HBEGF} \mathrm{Over}{ }^{\mathrm{Col} 2}$ cartilage grew much quicker than those from $W T$ (Fig. 2I) and were resistant to tumor necrosis factor $-\alpha(\mathrm{TNF} \alpha)$-induced apoptosis (Fig. 2J). Meanwhile, synovial mesenchymal progenitors were not affected (fig. S5). Together, our in vivo and in vitro data demonstrate that HBEGF overexpression produces more chondroprogenitors in articular cartilage with superior proliferation and survival abilities.

When subjected to chondrogenic differentiation, progenitors from HBEGF Over ${ }^{\mathrm{Col} 2}$ cartilage expressed more Prg4 but less cartilage matrix (Aggrecan, Col2a1, and Col10a1) and protease (Mmp13) markers (Fig. $2 \mathrm{~K}$ ). They differentiated into Alcian blue-positive cartilage, albeit the staining intensity was less than $W T$ (Fig. 2L). These in vitro data indicate that overexpression of HBEGF modestly decreases chondrogenic differentiation; however, immunostaining confirmed that proteoglycan, type II collagen, type X collagen, and MMP13 (fig. S6) amounts were not altered in HBEGF Over ${ }^{\text {Col2 }}$ cartilage, suggesting that overactivation of EGFR signaling does not negatively affect cartilage components in vivo.

As a transmembrane protein, HBEGF is cleaved by a sheddase and released from the cell membrane for paracrine and systemic actions (21). Because EGFR is important for the development and homeostasis of multiple organs, a concern is raised about possible side effects of constitutively expressing HBEGF. However, we did not observe p-EGFR in major organs, such as heart, liver, spleen, lung, kidney, and brain from adult $\mathrm{HBEGF}$ Over ${ }^{\mathrm{Col} 2}$ mice (fig. S7A). Endogenous EGFR and HBEGF were also not altered (fig. S7A). The morphology of these vital organs remained the same as WT mice at 5 and 12 months of age (fig. S7B), indicating no substantial side effects of cartilage-specific HBEGF overexpression.

\section{EGFR overactivation attenuates OA progression}

Next, we sought to study the effect of HBEGF overexpression on OA progression induced by surgical destabilization of the medial meniscus (DMM) (Fig. 3A). Two months after surgery, WT knees started to lose proteoglycan and exhibit fibrillation at the cartilage surface (Fig. 3, B and C). At 4 months after surgery, WT knees displayed severe cartilage erosion beyond the tidemark, accompanied by uneven cartilage surface or clefts. In contrast, knees in HBEGF Over ${ }^{\mathrm{Col} 2}$ mice showed only a minor loss in proteoglycan content 2 months after DMM surgery. Two months later, the articular surface was still intact, although cartilage was thinner than that in sham knees. Quantifying OA severity at 4 months after surgery revealed that overexpression of HBEGF reduced Mankin score from 10.0 to 2.1 at this stage (Fig. 3C). These data provided in vivo evidence that overactivation of EGFR could protect cartilage from degeneration upon $\mathrm{OA}$-inducing insults.

To eliminate the developmental effect in HBEGF Over ${ }^{\mathrm{Col} 2}$ mice, we next constructed an inducible model Aggrecan-CreER DTR (HBEGF Over ${ }^{A g c E R}$ ). These mice had normal articular cartilage before injury since tamoxifen was injected immediately before DMM surgery (Fig. 3D). IHC confirmed higher amounts of HBEGF and p-EGFR in articular cartilage of HBEGF Over ${ }^{\text {AgcER }}$ compared to the sham knees 1 month after induction (fig. S8). Four months after DMM surgery, whereas WT mice developed late OA with most cartilage eroded, HBEGF Over ${ }^{A g c E R}$ maintained relatively intact articular cartilage with a low Mankin score of 2.5 (Fig. 3, E and F). Our prior study demonstrated that nanoindentation of the cartilage surface is a sensitive method to detect early $\mathrm{OA}$ in mice (22). In line with this, the surface indentation modulus $E_{\text {ind }}$ was drastically reduced by $67 \%$ in $W T$ cartilage at 1 month after DMM but remained unchanged in HBEGF Over ${ }^{A g c E R}$ mouse knees (Fig. 3G), suggesting that overexpressing HBEGF in adult cartilage preserves the mechanical functions of the cartilage surface after OA injury.

HBEGF binds and signals through EGFR as well as another EGFR family member, ErbB4 (23). To study whether EGFR mediates the action of HBEGF on cartilage in vivo, we treated HBEGF Over ${ }^{A g c E R}$ mice and WT controls with the EGFR-specific inhibitor gefitinib (24) once every other day for 2 months after tamoxifen induction and DMM surgery. Similar to our previous data (25), gefitinib moderately accelerated OA progression in WT DMM knees, increasing Mankin score from 6.5 to 9.8 (Fig. 4, A and B). Gefitinib completely abolished the protective effect of HBEGF Over ${ }^{A g c E R}$ on articular cartilage after DMM surgery, leading to marked cartilage erosion with an elevated Mankin score of 9.0. DMM mainly reduced the thickness of uncalcified cartilage, resulting in 38 and $74 \%$ decreases in vehicle- and gefitinib-treated WT mice, respectively (Fig. 4C). In HBEGF Over ${ }^{\text {AgcER }}$ mice, although DMM alone did not alter the cartilage thickness, cotreatment with gefitinib greatly lessened the thicknesses of uncalcified and total cartilage by 75 and $59 \%$, respectively (Fig. 4C). Furthermore, the von Frey behavioral pain test indicated that HBEGF Over ${ }^{A g c E R}$ mice developed a similar degree of pain as $W T$ mice at 1 week after DMM but quickly recovered to baseline as sham mice, suggesting that overexpressing HBEGF also has functional benefits (Fig. 4D). However, gefitinib abolished this effect. We did not detect any effect of this inhibitor on sham knees from $W T$ or $H B E G F$ Over ${ }^{\text {AgcER }}$ mice. Therefore, our results indicated that HBEGF signals through EGFR in vivo to execute its chondroprotection action. 
A

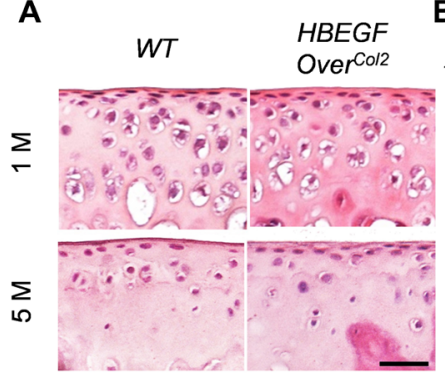

B

C

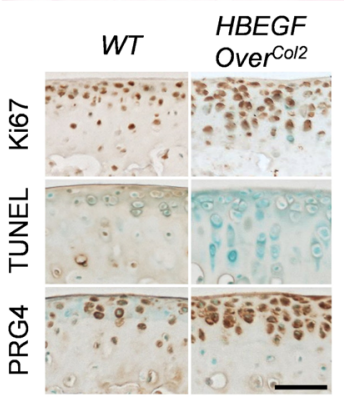

$\mathbf{F}$

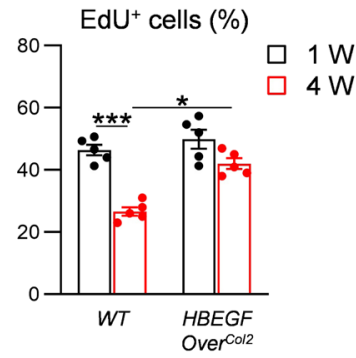

G
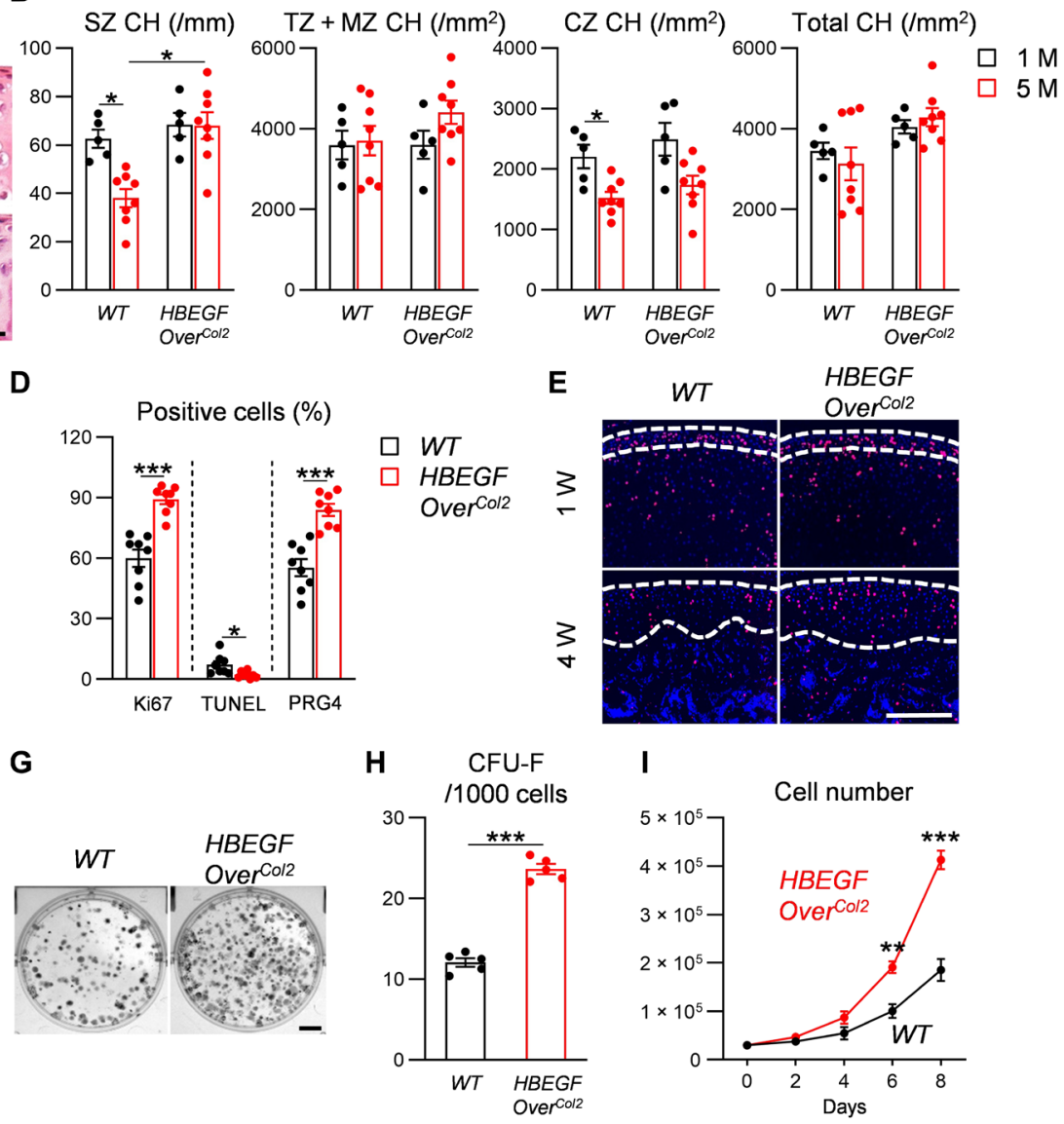

J

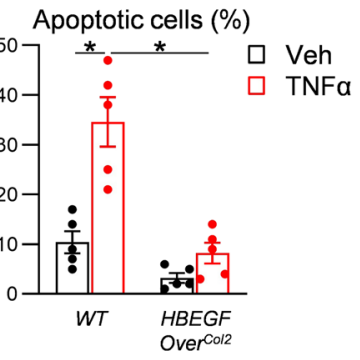

K

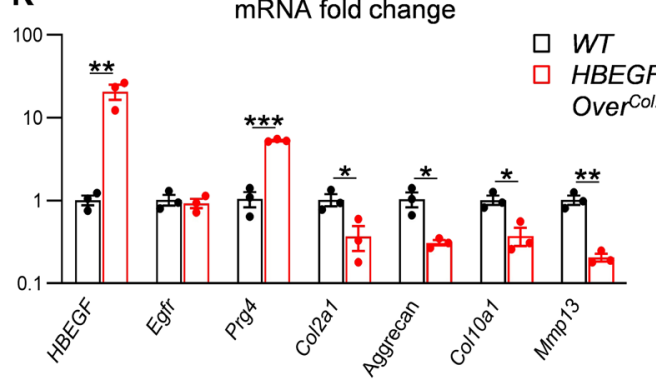

$\mathbf{L}$

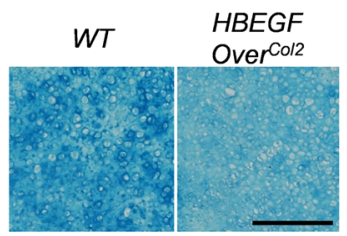

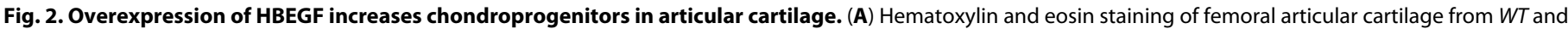

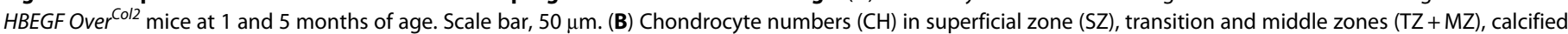

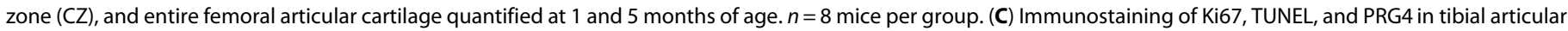

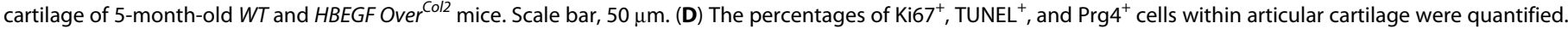

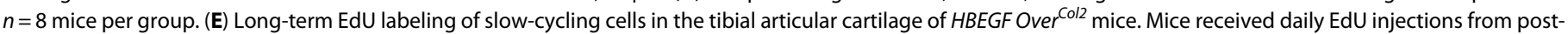

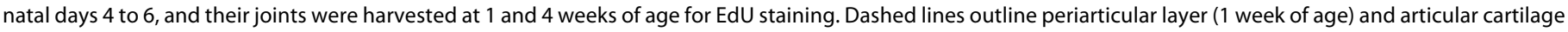

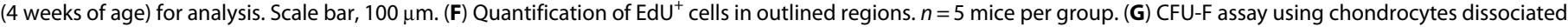

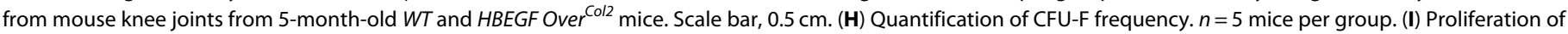

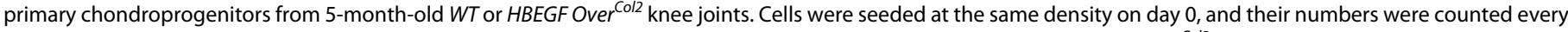

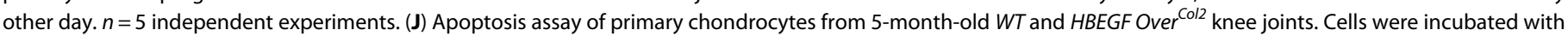

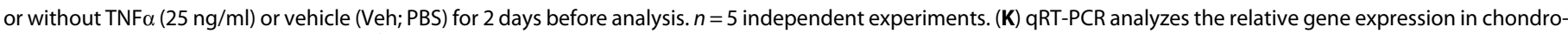

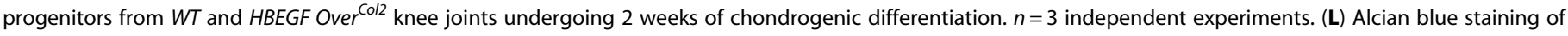

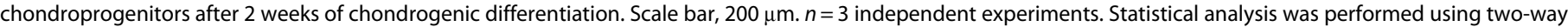

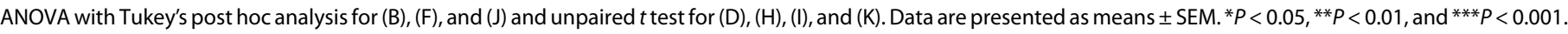

\section{Synthesis and characterization of TGF $\alpha$-NPs}

Cartilage expresses TGF $\alpha$ and other EGFR ligands (8); however, the above mouse studies indicated that endogenous EGFR ligand ex- pression is not sufficient to protect cartilage against OA. To activate EGFR for OA treatment, we chose one of the most potent EGFR ligands, TGF $\alpha$ (26), as an activator. However, TGF $\alpha$ is not stable in 

Col2-Cre HBEGF

D
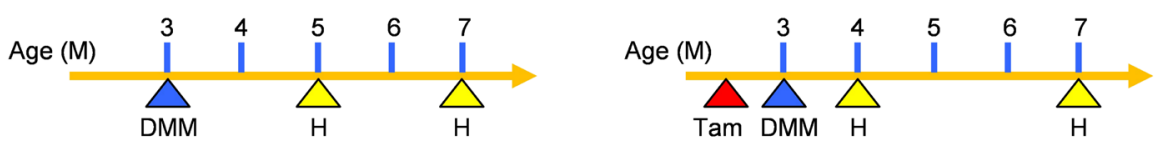

B

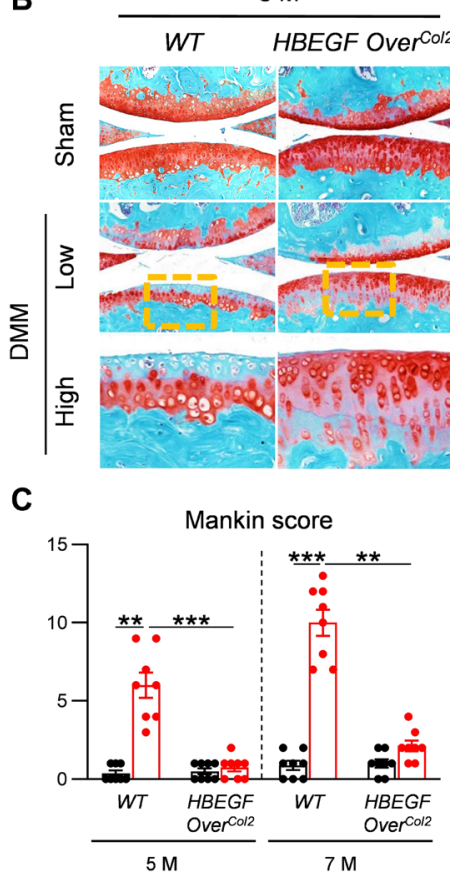

$\mathbf{F}$

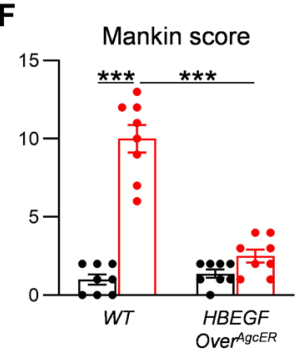

E

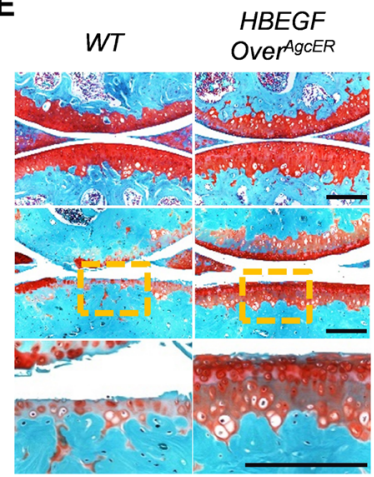

Agc-CreER HBEGF

G

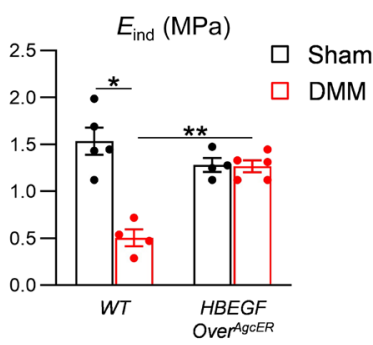

Fig. 3. Overexpressing HBEGF in articular cartilage delays OA progression. (A) Schematic showing the study protocol of WT and HBEGF Over ${ }^{\mathrm{CO} / 2}$ mice with DMM surgery at 3 months of age. $\mathrm{H}$ indicates time points when histology was performed. (B) Safranin O/Fast Green staining of DMM and sham joints at the medial site from 5-and 7-month-old WT and HBEGF Over ${ }^{\text {Col2 }}$ mice. Low: low-magnification image; high: high-magnification image of the yellow boxed area above. Scale bars, $200 \mu \mathrm{m}$. (C) The OA severity was measured by Mankin score. $n=8$ mice per group. (D) Schematic showing the study protocol of WT and HBEGF Over ${ }^{A g c E R}$ mice with tamoxifen (Tam) injections and DMM surgery at 3 months of age. (E) Safranin O/Fast Green staining of WT and HBEGF Over ${ }^{A g c E R}$ DMM and sham joints at the medial site from mice 7 months of age. Scale bars, $200 \mu \mathrm{m}$. (F) The OA severity was measured by Mankin score. $n=8$ mice per group. (G) Nanoindentation assay was performed on the femoral cartilage surface at 1 month after surgery. $E_{\text {ind }}$ modulus. $n=4$ to 5 mice per group. Statistical analysis was performed using two-way ANOVA with Tukey's post hoc analysis. Data are presented as means \pm SEM. ${ }^{*} P<0.05,{ }^{* *} P<0.01$, and ${ }^{* * *} P<0.001$.

the circulation, and direct injection of it into knee joints suffers from rapid clearance due to its low molecular weight $(5.6 \mathrm{kDa})$. To overcome this challenge, we engineered an NP delivery system to prolong the retention of active TGF $\alpha$ in the knee joint. Briefly, bacteria-expressed human TGF $\alpha$ were synthesized and site-specifically labeled at the $\mathrm{C}$ terminus with a constrained alkyne, dibenzocyclooctyne (DBCO), via sortase-tag-expressed protein ligation $(27,28)$. TGF $\alpha$-NPs were then prepared via copper-free click chemistry, by simply mixing TGF $\alpha$-DBCO with azide-functionalized NPs (Fig. 5A). Azide-functionalized NPs were made from 55 mole percent (mol \%) poly(ethylene glycol)-polycaprolactone (PEG-PCL)/ $20 \mathrm{~mol} \%$ poly[L-lysine-block-poly(e-caprolactone)] (PLL-PCL)/ 25 mol \% 1,2-distearoyl-sn-glycero-3-phosphoethanolamine- $N$ [azido(polyethylene glycol)-5000] (DSPE-PEG5K-N $\mathrm{N}_{3}$ ) using the film hydration method.

TGF $\alpha$-NPs resembled spheres with a hydrodynamic diameter of $25.93 \mathrm{~nm}$ (Fig. 5B). Because NP surface charge could be adjusted to augment the interaction between the therapeutic agents and the anionic glycosaminoglycans in the cartilage, the cationic diblock copolymer PLLPCL was synthesized (fig. S9) and introduced into the PEG-PCL NPs to reduce their surface charge from -4.2 to $-1 \mathrm{mV}$ (Fig. 5C). After the conjugation of TGF $\alpha$, the surface charge of TGF $\alpha$-NPs became more negative, mostly due to the negatively charged TGF $\alpha$. However, the magnitude of the surface charge of TGF $\alpha$ NPs was still reduced in NPs containing PLL-PCL. For example, the surface charges of TGF $\alpha$-NPs in the presence and absence of PLL-PCL were -13.7 and $-19.4 \mathrm{mV}$, respectively. We next characterized stability, cytotoxicity, and specificity of newly synthesized TGF $\alpha$-NPs. We did not detect any change in the hydrodynamic diameter of TGF $\alpha$-NPs in water for at least 1 week (Fig. 5D) or in bovine synovial fluid for 24 hours (Fig. 5E). MTT [3-(4,5-dimethylthiazol-2-yl)-2,5diphenyltetrazolium bromide] assay showed that TGF $\alpha$-NP treatment (up to $10 \mu \mathrm{M}$ TGF $\alpha$ content) for 24 hours did not affect the viability of mouse primary chondrocytes (Fig. 5F). Western blot demonstrated that TGF $\alpha$-NPs activated EGFR downstream target ERK as potently as free TGF $\alpha$, whereas Ctrl-NPs (NP with no TGF $\alpha$ conjugation) had no such effect (Fig. 5, G and $\mathrm{H}$ ). In addition, similar to free TGF $\alpha$, TGF $\alpha$-NPs changed chondrocytes from a polygonal cell shape to a more spindle cell shape after 2 days of treatment (fig. S10). Using fluorescent rhodamine-labeled TGF $\alpha$-NPs, we found that TGF $\alpha$-NPs bound to the surface of primary chondrocytes in a TGF $\alpha$-specific manner (Fig. 5I). Moreover, in human articular cartilage explants, TGF $\alpha$-NPs activated ERK (fig. S11A); reduced the expression of chondrocyte markers Sox9, Aggrecan, and Col2a1; and elevated the expression of lubricant Prg4, cyclin gene Ccnd1, and proteinase MMP13 (fig. S11B). These results are consistent with previous studies reporting the effect of TGF $\alpha$ on rodent and bovine cartilage explants (29). TGF $\alpha$-NPs decreased the expression of Adamts5, the major aggrecanase, in human cartilage, which is also in line with a previous report studying TGF $\alpha$ actions on rat chondrocytes (30). Together, our data demonstrate that TGF $\alpha$-NPs are stable, nontoxic, and functional.

\section{TGF $\alpha$-NP cartilage uptake, penetration, and joint retention}

Human knee articular cartilage is about 2 to $4 \mathrm{~mm}$ thick, and the superficial layer makes up 10 to $20 \%$ of cartilage thickness (31). To increase cartilage retention and to penetrate deeper into the cartilage, we doped cationic PLL-PCL into the NPs to reduce their surface charge. Using a near-infrared fluorescence probe IRDye $800 \mathrm{CW}$ as 
A

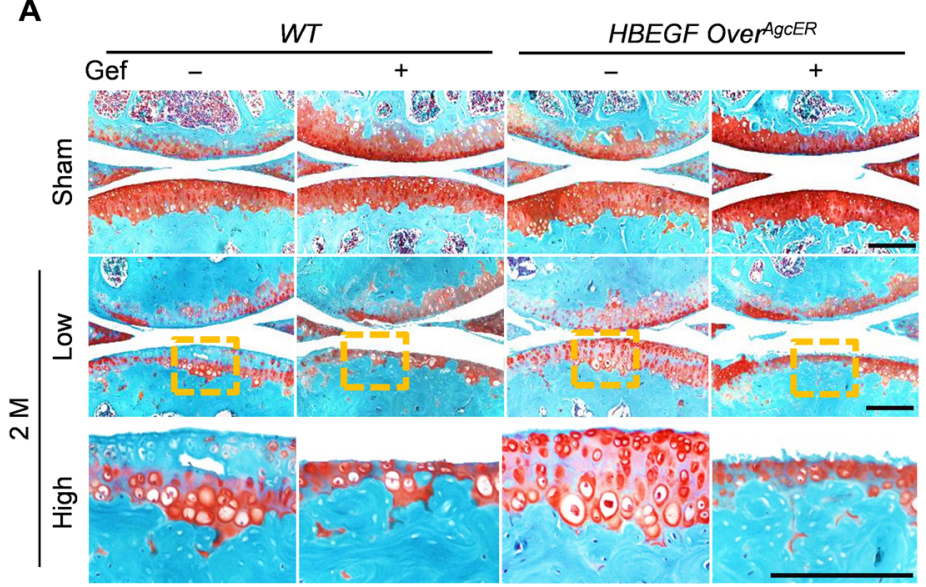

D

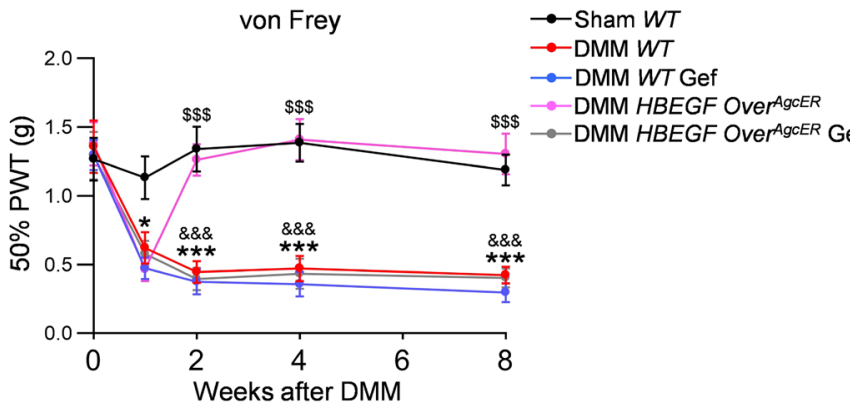

B

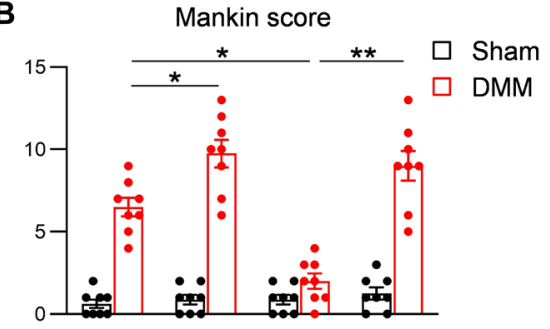

C
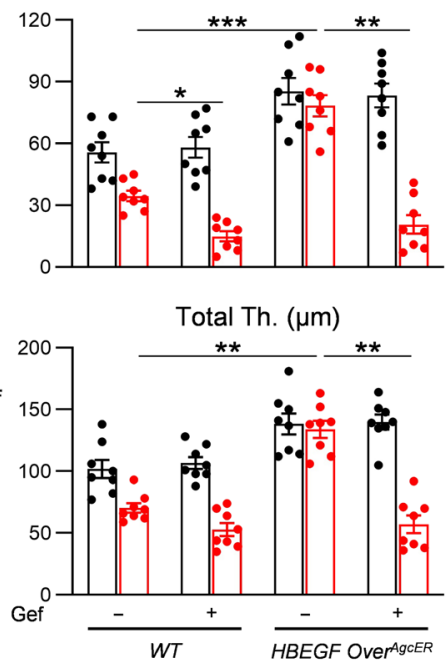

Fig. 4. The protective action of HBEGF overexpression on articular cartilage during OA development is EGFR dependent. (A) Safranin O/Fast Green staining of vehicle- and gefinitib (Gef)-treated WT and HBEGF Over ${ }^{A g C E R}$ knee joints at the medial site 2 months after surgery. Low: low-magnification image; high: high-magnification image of the yellow boxed area above. Scale bars, $200 \mu \mathrm{m}$. (B) The OA severity was measured by Mankin score. $n=8$ mice per group. (C) Average thicknesses of uncalcified (Uncal. Th.) and total (Total Th.) cartilage quantified at 2 months after surgery. $n=8$ mice per group. (D) von Frey assay was performed at $0,1,2,4$, and 8 weeks after surgery. PWT, paw withdrawal threshold. $n=8$ mice per group. Statistical analysis was performed using one-way ANOVA with Tukey's post hoc analysis for (D) and two-way ANOVA with Tukey's post hoc analysis for (B) and (C). Data are presented as means \pm SEM. ${ }^{*} P<0.05,{ }^{*} P<0.01$, and ${ }^{* * *} P<0.001$ in (B) and (C). ${ }^{*} P<0.05$ and ${ }^{* * *} P<0.001$ for DMM $W T$ versus sham $W T ;{ }^{\$ \$ \$} P<0.001$ for DMM HBEGF Over ${ }^{A g C E R}$ versus DMM $W T ;{ }^{*} \& \& P<0.001$ for DMM HBEGF Over ${ }^{A g c E R}$ versus DMM HBEGF Over ${ }^{A g c E R}$ Gef in (D).

a label, we found that bovine cartilage explants took up much more PLL-PCL-doped TGFo-NPs than non-PLL-PCL-doped TGFo-NPs or TGFo-DBCO after a 24-hour incubation (fig. S12). To study penetration, we labeled TGF $\alpha-\mathrm{NPs}$ and TGF $\alpha$-DBCO with rhodamine. TGF $\alpha$-DBCO and non-PLL-PCL-doped TGF $\alpha$-NPs only accumulated at the cartilage surface but did not penetrate deep inside the cartilage over a 6-day culture period (Fig. 6, A and B, and fig. S13, A to D). PLL-PCL-doped TGF $\alpha$-NPs efficiently bound to the surface of bovine cartilage explants at day 2 and gradually penetrated inside by at least $1 \mathrm{~mm}$ by day 6 (Fig. 6, A and B, and fig. S13, E and F). Quantitative analysis of the fluorescence images revealed that PLLPCL-doped TGFo-NPs exhibited more than a 4.76-fold improvement in cartilage penetration at day 6 compared with non-PLL-PCLdoped TGFo-NPs (Fig. 6C) with an estimated diffusivity of $1.1 \mu \mathrm{m}^{2} / \mathrm{s}$. This result demonstrated the improved cartilage penetration and accumulation of TGF $\alpha$-NPs with PLL-PCL.

Next, we directly injected TGF $\alpha$-NPs or TGF $\alpha$-DBCO labeled with IRDye $800 \mathrm{CW}$ into the knee joint to study retention under healthy and OA conditions (Fig. 6D). DMM was performed on the joints 2 months before injection to mimic OA. After a single injection, the fluorescence signal in joints injected with TGF $\alpha$-NPs was much higher than those injected with TGF $\alpha-\mathrm{DBCO}$ at all time points, indicating the increased retention of TGF $\alpha$-NPs (Fig. 6E). Quantitative analysis of fluorescence images showed that TGF $\alpha$-NPs in OA joints were retained even longer than those in healthy joints (Fig. 6F).

We also examined the biodistribution of TGF $\alpha-\mathrm{NPs}$ in internal organs, blood, and joint components. At 24 hours after injection, fluorescent signals were detected on the cartilage surfaces of the patella, femoral condyles, and the tibial plateau, as well as on meniscus (fig. S14, A and B). TGF $\alpha$-NPs were mainly accumulated in liver and kidneys, but no signal was detected in the blood, heart, and spleen, indicating that NPs can be cleared quickly from circulation. One month later, there were no TGF $\alpha$-NPs left in liver and kidney (fig. S14, C and D).

\section{TGF $\alpha$-NP rescue of OA cartilage degeneration after DMM surgery}

To test their therapeutic effect on OA, we injected TGF $\alpha$-NPs into mouse knee joints after DMM once every 3 weeks. Control groups include knee joints injected with phosphate-buffered saline (PBS), TGF $\alpha$-DBCO, and Ctrl-NPs (NP with no TGF $\alpha$ conjugation). In line with our previous findings (8), EGFR activity, as indicated by p-EGFR, was decreased in PBS-treated articular cartilage after DMM (Fig. 7A). Injections of TGF $\alpha-N P s$, but not TGF $\alpha-D B C O$ or Ctrl-NPs, successfully elevated cartilage EGFR activity to that of the sham group (Fig. 7A). At 2 and 3 months after surgery, both the 
A
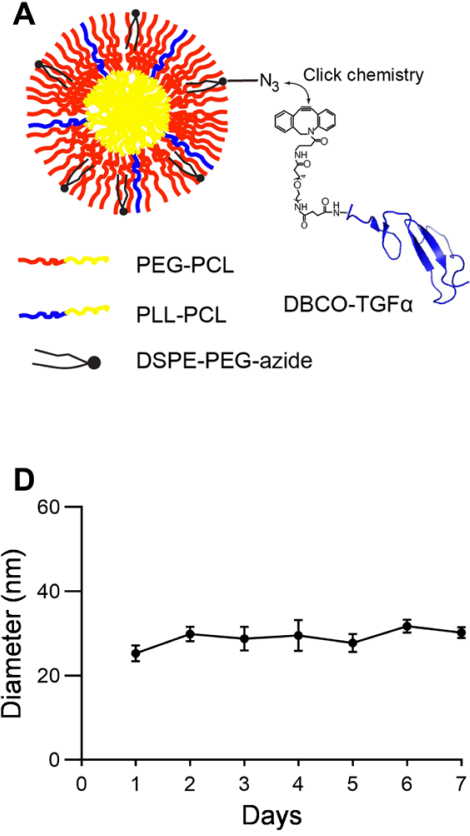

G

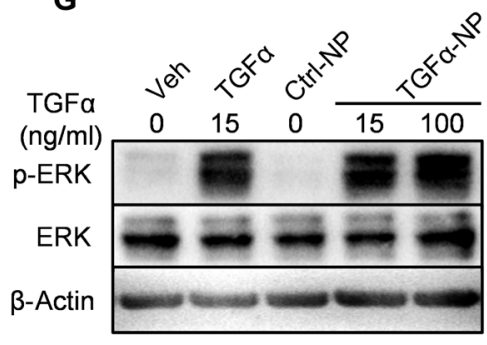

B

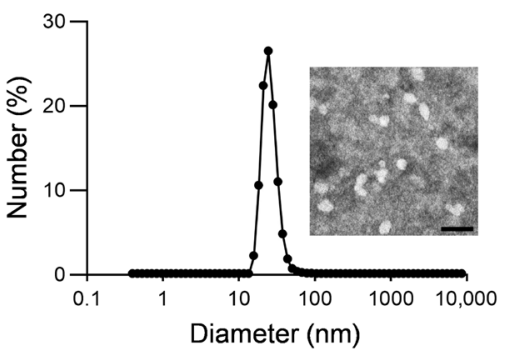

E

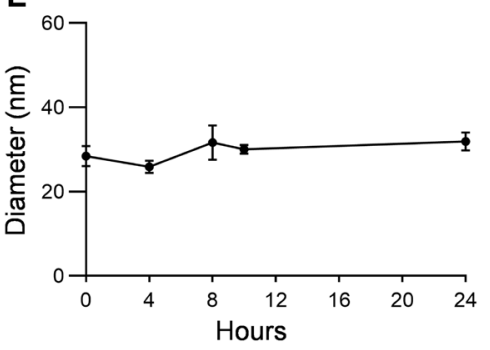

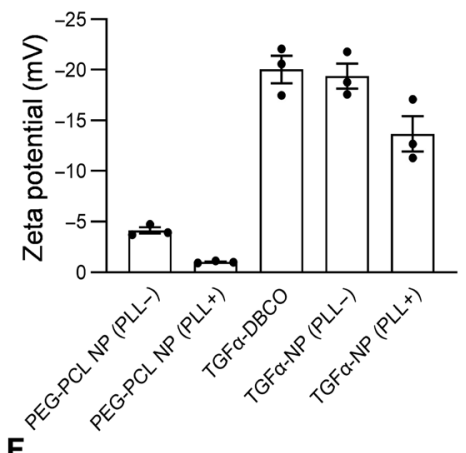

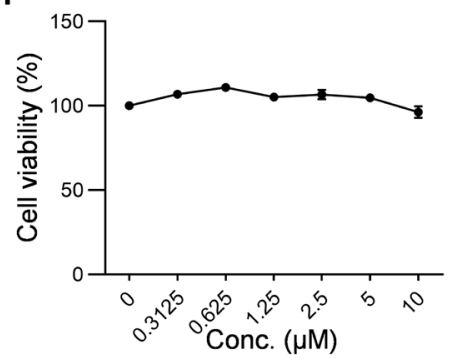

I

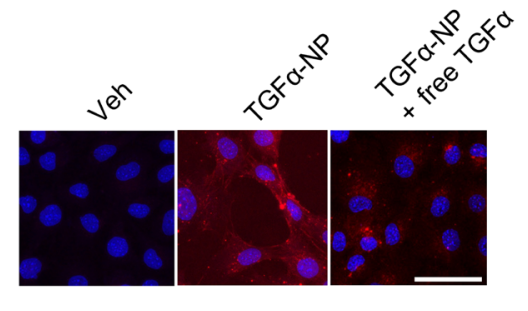

H

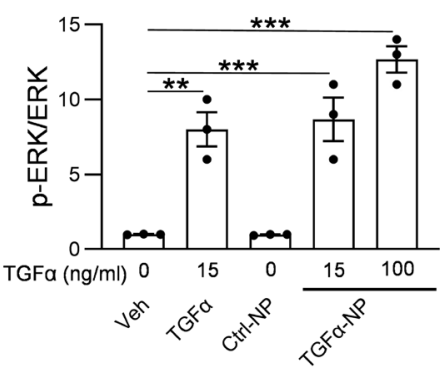

Fig. 5. Preparation and characterization of TGF $\alpha$-NPs. (A) Schematic diagram of TGF $\alpha$-NPs. TGF $\alpha$-NPs were prepared by conjugating TGF $\alpha$ onto polymeric micellar NPs via copper-free click chemistry. (B) Dynamic light scattering (DLS) measurements of TGF $\alpha$-NP hydrodynamic diameter (size) and representative image of TGF $\alpha$-NPs examined by transmission electron microscopy. Scale bar, $100 \mathrm{~nm}$. (C) Zeta potential measurements of TGF $\alpha$-DBCO, PEG-PCL NPs with or without PLL-PCL, and TGF $\alpha$-NPs with or without PLL-PCL in $0.1 \times$ PBS (pH 7.4). PLL+ denotes the NPs that contain PLL-PCL, and PLL- denotes the NPs that do not contain PLL-PCL. $n=3$ independent experiments. (D) Stability of TGF $\alpha$-NPs in water was evaluated by monitoring DLS measurement of TGF $\alpha$-NP hydrodynamic diameter for up to 7 days. $n=3$ independent experiments. (E) Stability of TGF $\alpha$-NPs in bovine synovial fluid of knee joint was evaluated by monitoring DLS measurement of TGF $\alpha$-NP hydrodynamic diameter for up to 24 hours. $n=3$ independent experiments. (F) Cell viability of primary mouse chondrocytes after incubation with TGF $\alpha$-NPs at different concentrations. $n=3$ independent experiments. (G) Western blot of EGFR downstream signal (p-ERK) in articular cartilage chondrocytes treated by vehicle (PBS), free TGF $\alpha$ (15 ng/ml), Ctrl-NPs (i.e., no TGF $\alpha$ conjugation), or TGF $\alpha$-NPs (15 or $100 \mathrm{ng} / \mathrm{ml}$ of TGF $\alpha$ content). (H) Quantitative analysis of the relative protein amount (p-ERK/ERK) based on the images of Western blot as in (G). $n=3$ independent experiments. (I) Confocal images of mouse primary chondrocytes treated with vehicle (PBS), TGF $\alpha$-NPs (10 nM TGF $\alpha$ content), or TGF $\alpha$-NPs (10 $\mathrm{nM}$ TGF $\alpha$ content) in the presence of free TGF $\alpha(100 \mu \mathrm{g} / \mathrm{ml})$. Scale bar, $50 \mu \mathrm{m} . n=3$ independent experiments. Statistical analysis was performed using one-way ANOVA with Dunnett's post hoc analysis. Data are presented as means \pm SEM. ${ }^{* *} P<0.01$ and ${ }^{* * *} P<0.001$.

TGF $\alpha$-DBCO group and Ctrl-NP group displayed a similar pattern of cartilage degeneration, including erosion and surface fibrillation, similar to the PBS group (Fig. 7B). Mankin scores of these three control groups at 3 months after DMM were similarly around 8.5 (Fig. 7C), mainly due to the reduction of uncalcified cartilage thickness (Fig. 7D). Knee joints in the TGF $\alpha$-NP group maintained cartilage integrity at 2 months after DMM and displayed minor signs of degeneration at 3 months after DMM (Fig. 7B). The Mankin scores at both 2 and 3 months were markedly decreased compared to control groups and their uncalcified zones were largely preserved (Fig. 7, C and D, and fig. S15). Similar results were also observed when TGF $\alpha$-NP injections started 1 month after DMM (to allow early OA develop) (fig. S16).
To understand the mechanism underlying the therapeutic effect of EGFR overactivation, we performed IHC of EGFR downstream targets in sham and DMM knees at 2 months after surgery of HBEGF Over ${ }^{\mathrm{Col} 2}$ mice and TGF $\alpha$-NP-injected WT mice (fig. S17). Consistent with the cartilage degeneration phenotype, DMM reduced the amounts of anabolic factors, such as p-ERK and PRG4, and increased the amounts of catabolic factors, such as MMP13 and ADAMTS5, in the articular cartilage. Overexpression of HBEGF or intra-articular injection of TGF $\alpha$-NP efficiently reversed those changes, resulting in similar staining patterns of those proteins between sham and DMM knees of these mice.

Subchondral bone sclerosis is a late OA symptom. Our previous study established a three-dimensional micro-computed tomography (3D 
A

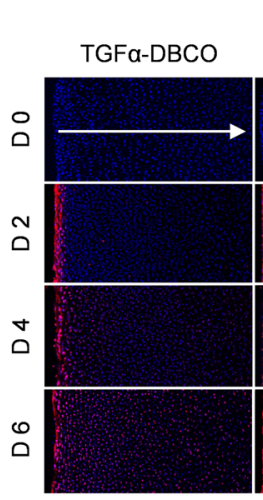

C

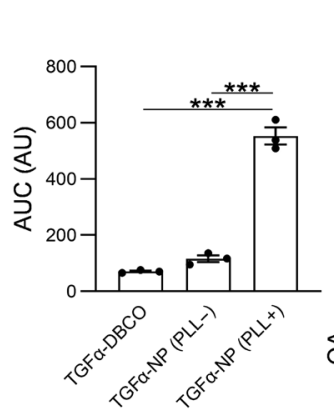

E

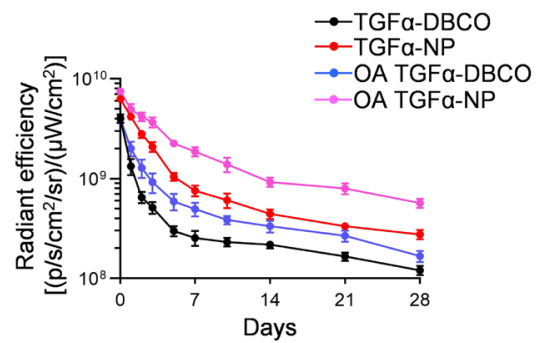

B

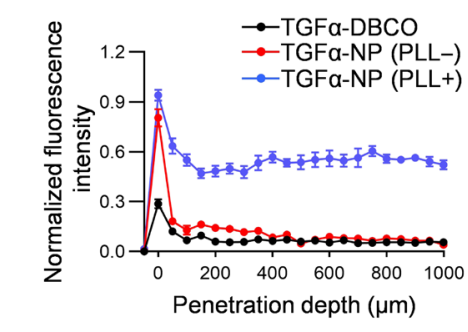

D

$\begin{array}{cc}\text { TGFa-NP } & \text { TGFa-NP } \\ (\text { PLL-) } & (P L L+)\end{array}$

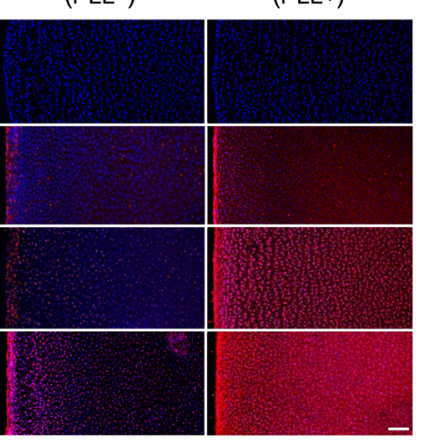

Days: $\begin{array}{lll}0 & 1 & 2\end{array}$ $\begin{array}{lllll}5 & 7 & 14 & 21 & 28\end{array}$

Radiant

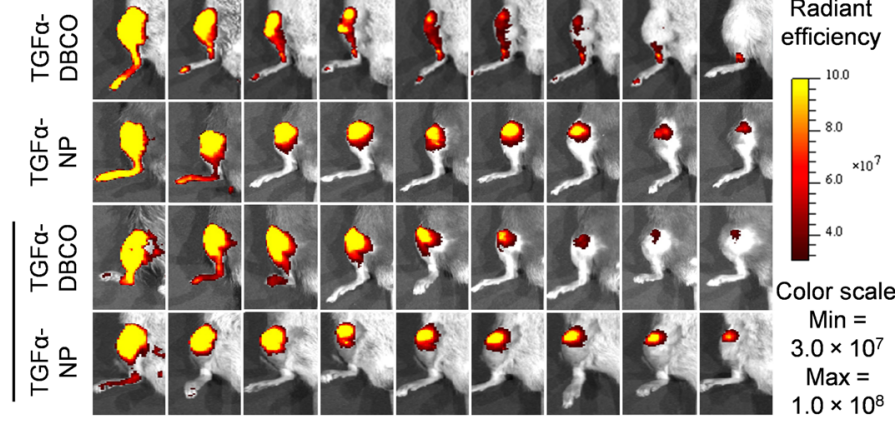

$\mathbf{F}$

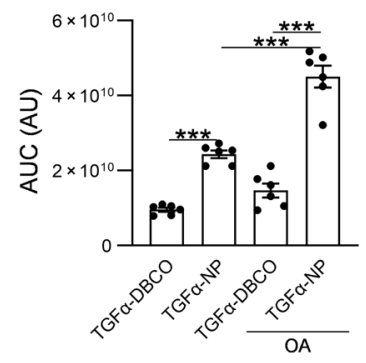

Fig. 6. TGF $\alpha$-NPs exhibit full-length penetration of human-thickness bovine articular cartilage and extend residence time in both healthy and diseased knee joints. (A) Representative confocal microscopy images of a cross section of bovine cartilage explants incubated with rhodamine-labeled TGF $\alpha$-NPs with or without PLL-PCL or free TGF $\alpha$ for 2, 4, and 6 days. Arrow indicates the diffusion direction. Scale bar, $200 \mu \mathrm{m}$. (B) Quantitative analysis of TGF $\alpha$-NP penetration depth into bovine cartilage explants after 6-day incubation. $n=3$ per group. (C) Quantitative analysis of area under the curve (AUC) based on fluorescence intensity profiles in (B). $n=3$ per group. (D) Representative fluorescence images of healthy and OA mouse knee joints over 28 days after intra-articular injection of IRDye $800 \mathrm{CW}$-labeled TGF $\alpha$ or TGF $\alpha$-NPs. (E) Quantitative analysis of time course fluorescence radiant efficiency within knee joints after intra-articular injection of IRDye $800 \mathrm{CW}$-labeled TGF $\alpha$ or TGF $\alpha$-NPs. $n=6$ per group. (F) Quantitative analysis of area under the curve based on fluorescence intensity profile in (E). $n=6$ per group. Statistical analysis was performed using one-way ANOVA with Tukey's post hoc analysis. Data are presented as means \pm SEM. ${ }^{* * *} P<0.001$. $\mathrm{AU}$, arbitrary units.

microCT) approach to accurately measure the thickness of SBP (9). Using this method, we confirmed that SBP thicknesses were elevated in PBS-treated, TGF $\alpha$-DBCO-treated, and Ctrl-NP-treated DMM knees relative to sham (Fig. 7, E and F). However, this increase was abolished in TGF $\alpha$-NP-treated DMM knees. Synovitis is another sign of OA (32). We observed thickening of the synovial lining layer and increased synovitis scores in DMM knees with PBS, TGF $\alpha$-DBCO, or Ctrl-NP treatment but not with TGF $\alpha$-NP treatment relative to sham (Fig. 7, G and H). Moreover, von Frey assay revealed that TGF $\alpha$-NP treatment attenuated OA-induced pain starting from

2 weeks after surgery (Fig. 7I). Together, these results demonstrate a therapeutic effect of intra-articular delivery of EGFR ligand-conjugated polymeric micellar NP.

Last, we examined whether 2 months of intra-articular injections of TGF $\alpha$-NPs caused any side effects to several major internal organs or to overall joint structure in WT mice. As shown in fig. S18A, we did not detect any obvious morphologic changes in heart, liver, spleen, lung, kidney, and brain between PBS-treated and TGF $\alpha$-NP-treated mice. Western blots indicated no change in EGFR activity in those organs after TGFo-NP injections (fig. S18B). Liver and lung had the highest expression of EGFR and $\mathrm{TGF} \alpha$, which were not affected by TGF $\alpha$-NP injections. Furthermore, the gross morphology of knee joints was not altered by 2 months of TGF $\alpha$-NP treatment (fig. S18C).

\section{DISCUSSION}

Previous studies from our group and others have demonstrated the important role of EGFR signaling in the development of articular cartilage and in OA progression (29). EGFR signaling can execute both anabolic and catabolic actions on cartilage chondrocytes; however, differences exist regarding how this signaling pathway may be best modulated for OA treatment. In the present study, we first provide genetic evidence demonstrating that overactivation of EGFR signaling modestly thickens the articular cartilage and completely blocks OA progression after DMM surgery. Other joint tissues, such as bone, synovium, and meniscus, appeared normal in mice up to 12 months of age and showed no pathological OA changes, such as osteophytosis and subchondral bone sclerosis, suggesting that EGFR signaling could be precisely applied in vivo to fulfill its anabolic actions without inciting catabolic effects. We then provided proof-of-principle evidence that administration of TGF $\alpha$ into mouse joints using an advanced NP delivery system is effective in attenuating DMM-induced OA initiation and development.

One mechanism underlying the therapeutic action of EGFR signaling is that EGFR activation promotes the lubrication function of articular cartilage, particularly the expression of lubricant Prg4. In humans, loss-of-function PRG4 mutation results in camptodactylyarthropathy-coxa vara-pericarditis syndrome with precocious joint failure (33). Mice lacking Prg4 display early OA (34). On the contrary, intra-articular treatment with recombinant PRG4 prevented 
A

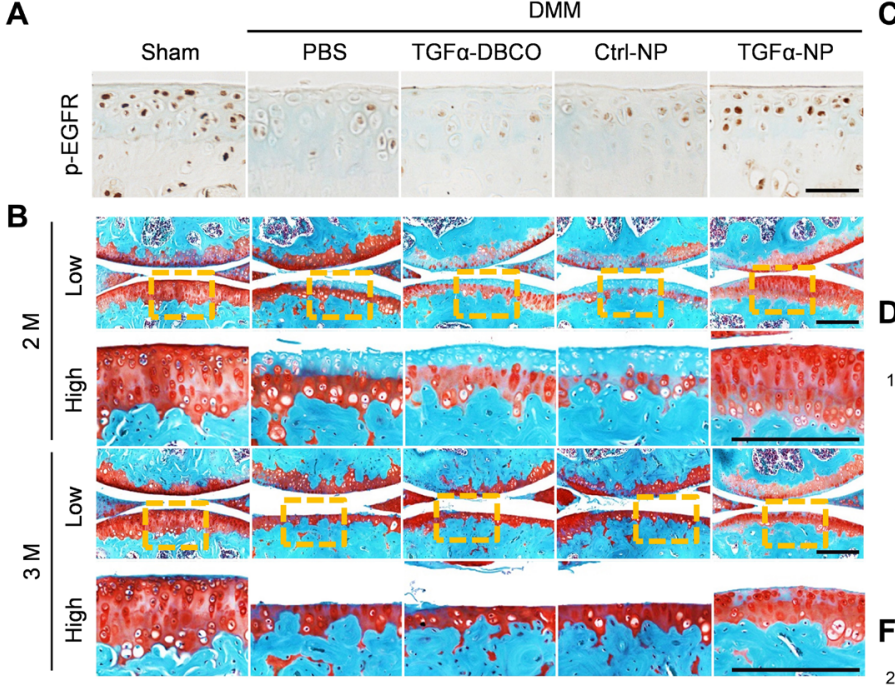

$\mathbf{E}$

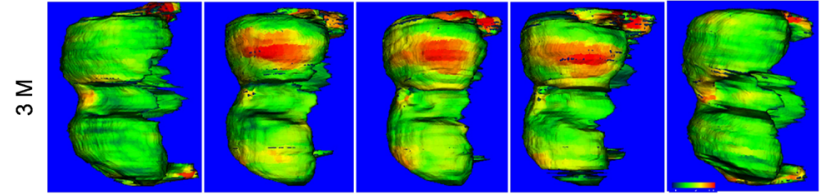

G

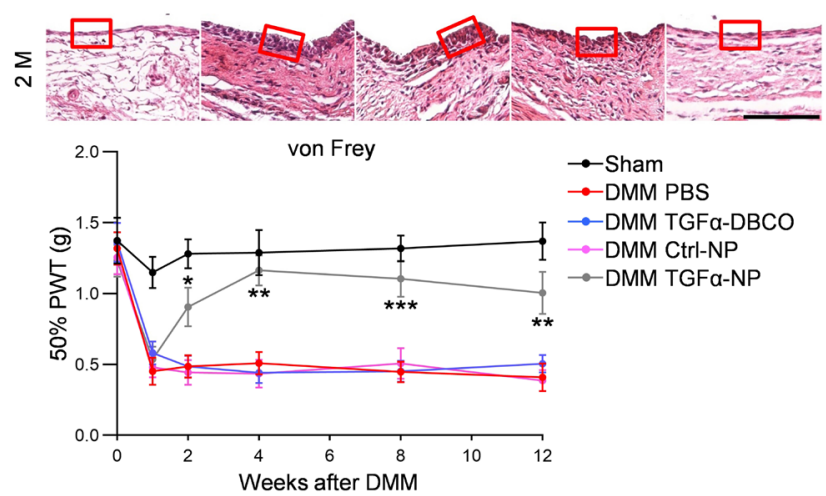

Fig. 7. TGF $\alpha$-NP treatment attenuates $O A$ progression after DMM surgery in mice. (A) Immunostaining of p-EGFR in mouse knee cartilage at 1 month after sham or DMM surgery. In the DMM group, mice were divided into four groups receiving PBS, TGF $\alpha$-DBCO, Ctrl-NP, or TGF $\alpha$-NP intra-articular treatments. Scale bar, $100 \mu \mathrm{m} . n=3$ mice per group. (B) Safranin O/Fast Green staining of knee joints at the medial site at 2 and 3 months after surgery. Low: low-magnification image; high: high-magnification image of the yellow boxed area above. Scale bars, $200 \mu \mathrm{m}$. (C) The OA severity of knee joints at 3 months after surgery measured by Mankin score. $n=8$ mice per group. (D) Average uncalcified (Uncal. Th.) cartilage thickness of knee joints at 3 months after surgery was quantified. $n=8$ mice per group. (E) Representative 3D color maps derived from microCT images showing SBP thickness (SBP Th.). Color ranges from 0 (blue) to $320 \mu \mathrm{m}$ (red). (F) SBP thickness at the medial posterior site of femoral condyle was calculated. $n=8$ mice per group. (G) Hematoxylin and eosin staining of mouse knee joints focusing on synovium at 2 months after surgery. Red boxed areas indicate the synovial tissues. Scale bar, $200 \mu \mathrm{m}$. (H) Synovitis score was measured. $n=8$ mice per group. (I) von Frey assay was performed at $0,1,2,4,8$, and 12 weeks after surgery. $n=8$ per group. Statistical analysis was performed using one-way ANOVA with Tukey's post hoc analysis. Data are presented as means \pm SEM. ${ }^{*} P<0.05,{ }^{* *} P<0.01$, and ${ }^{* * *} P<0.001$ in $(C),(D),(F)$, and $(H) .{ }^{*} P<0.05,{ }^{* *} P<0.01$, and ${ }^{* * *} P<0.001$ for DMM TGF $\alpha$-NP versus DMM PBS in (I).

cartilage degeneration in a rat OA surgery model (35). Delayed administration of teriparatide (Forteo), the recombinant human parathyroid hormone (1-34), was shown to have a chondro-regenerative effect, which correlated with increased Prg4 mRNA in mouse OA articular chondrocytes (36). In our previous study, we demonstrated that EGFR deficiency leads to reduced PRG4 amount in the articular cartilage and that TGF $\alpha$ promotes $\operatorname{Prg} 4$ expression in bovine cartilage (8). In the current study, we found that EGFR overactivation, either by overexpressing HBEGF or administration of TGF $\alpha$-NPs, preserved PRG4 in articular cartilage, which is otherwise diminished in OA. Together, we believe that PRG4 plays a critical role in mediating the chondroprotective action of EGFR signaling.

Currently, there are no disease-modifying drugs clinically approved for treating OA. Nonsteroidal anti-inflammatory agents have often been used for the short-term management of the pain symptoms in OA. More recently, several protein therapies, such as insulin-like growth factor (IGF) (37) and fibroblast growth factor 18 (38), have shown promise for OA treatment. However, intra-articular delivery of these therapeutic proteins has been largely limited by their rapid clearance from the joint space and their low penetration into the dense, avascular cartilage matrix. Consistent with this, we also observed that intra-articular injection of free TGF $\alpha$ had low joint retention and poor cartilage penetration and thus was ineffective in preventing OA development and progression. Because of its favorable pharmacokinetics, biodistribution, and specificity, NP-based drug delivery systems have been explored to improve drug delivery and therapeutic efficacy in OA treatment. For example, Geiger et al. (17) developed dendrimer-based nanocarriers to deliver IGF-1 to chondrocytes within joint cartilage. The dendrimer IGF-1 could penetrate full-thickness bovine cartilage and enhance the efficacy of IGF-1 in protecting both cartilage and bone in a rat surgical model of OA. Yan et al. (19) used NP-based small interfering RNA delivery to attenuate early inflammation in OA development.

We have addressed the TGF $\alpha$ delivery challenges by conjugating TGF $\alpha$ onto nanometer-sized polymeric micellar NPs. Polymeric micellar NPs are nanoscopic core/shell structures formed by amphiphilic block copolymers (39-41). Compared to other drug nanocarriers (42), the polymeric NPs provide several advantages, including their relatively small size and the use of similar formulations in different preclinical and clinical studies. In this work, polymeric micellar NPs were prepared from biocompatible and biodegradable polymers including PEG-PCL, PLL-PCL, and pegylated phospholipids. PEG, PCL, PLL, and phospholipids are clinically tested materials with well-characterized safety profiles. Moreover, the manufacture of 
these NPs is simple, reproducible, and scalable, which could allow fast translation into clinic use. We further used proximity-based sortase ligation to enable the highly efficient, site-specific bioconjugation of TGF $\alpha$ onto our NPs. Currently, one of the greatest challenges in combining protein-based targeting ligands with NPs is the low efficiency of bioconjugation. To address this limitation, we used a new strategy that uses a unique sortase fusion protein for the efficient and site-specific modification of the $C$ terminus of recombinant proteins. On the basis of this method, a DBCO moiety was ligated to the $\mathrm{C}$ terminus of TGF $\alpha$. The availability of the DBCO group, subsequently, allows for the facile bioconjugation of the TGF $\alpha$ to azide-labeled NPs using highly efficient click chemistry. Last, the TGF $\alpha$-NPs exhibit therapeutic efficacy, with no detectable side effects on joint structure and peripheral organs. The TGF $\alpha-N P s$ resolve the issues of short in vivo half-life and low cartilage penetration efficiency of free growth factors. Local delivery of TGF $\alpha$-NPs into knee joints after OA injury effectively attenuated cartilage degeneration and blocked SBP sclerosis and joint pain in mice, thus paving the way toward future translational studies that target EGFR signaling. We are aware that mouse articular cartilage is much thinner than the human counterpart. Although our culture experiment suggests that TGF $\alpha-\mathrm{NP}$ can penetrate at least $1 \mathrm{~mm}$ into bovine cartilage, future studies are needed to optimize the nanoformulation to achieve better penetration in large-animal models before translating this approach into clinical applications.

Activation of EGFR signaling for OA treatment has several concerns. It is well established that EGFR ligands, including TGF $\alpha$ and HBEGF, reduce anabolic gene expression (Sox9, Col2a1, and Aggrecan) and increase catabolic gene expression $(M m p 13)$ in cultured chondrocytes $(30,43)$. Our studies using chondroprogenitors derived from HBEGF Over ${ }^{\mathrm{Col} 2}$ articular cartilage showed decreased anabolic gene expression and Alcian blue staining when they underwent chondrogenic differentiation in vitro. However, these changes are relatively modest, and a considerable amount of cartilage matrix still remained in the pellet. Moreover, histology of adult HBEGF Over ${ }^{\mathrm{Col} 2}$ knees revealed no change in type II and type X collagen and proteoglycan amounts, indicating that cell culture data might not be directly correlated to animal data. We also observed that MMP13 expression was decreased in $\mathrm{HBEGF} \mathrm{Over}^{\mathrm{Col} 2}$ chondrogenic culture but unchanged in the HBEGF Over ${ }^{\text {Col2 }}$ joints. These data are contradictory to a previous report that adding HBEGF to chondrocyte culture increases $M m p 13$ expression (44). We reason that HBEGF dosage and in vivo versus in vitro conditions might determine the final outcomes.

Several EGFR activation mouse models have been investigated for OA study. Most of them used Mig6 knockout models, all having elevated EGFR activity in the cartilage. The global ablation of Mig6 causes severe joint deformity in young mice (45), whereas the cartilagespecific (Col2-Cre) and skeletal-specific (Prx1-Cre) knockout of Mig6 have much more minor joint phenotypes, with initial anabolic expansion of articular cartilage followed by modest degeneration or osteophyte formation at a later age $(12,13)$. Similar to our study, a previous report took advantage of DTR to construct Dermo1-Cre HBEGF (HBEGF Over ${ }^{\text {Dermol }}$ ) mice (46). At a young age, these mice develop chondrodysplasia, chondroma, OA-like joint defects, and bone phenotypes. While Dermo1-Cre broadly targets mesenchymal lineage cells (47), the Col2-Cre (48) and Aggrecan-CreER (49) are more specific for cartilage tissue. Li et al. (46) also reported that Col2-Cre HBEGF mice have normal body weight and body size but slightly distorted knee joints. In our hands, HBEGF Over ${ }^{C o l 2}$ and Over ${ }^{A g c E R}$ mice do not show any joint deformity and bone abnormalities. Therefore, we propose that the therapeutic effect of EGFR signaling depends on its activity and specificity. Mig6 global knockout and HBEGF Over ${ }^{\text {Dermo1 }}$ mice have the highest EGFR activity not only in cartilage but also in other organs, thus tipping the balance more toward catabolic actions on cartilage. Mig6 CKO mice have increased EGFR activity but not as high as the previous two models such that they exhibit anabolic actions first and then catabolic actions. In addition, we cannot rule out the possibility that Mig6 knockout mice have off-target effects because Mig6 also regulates signaling pathways other than EGFR, such as HGF/Met (50). Because of their cartilage and EGFR specificity, HBEGF Over ${ }^{\mathrm{Col} 2}$ and Over ${ }^{\text {AgcER }}$ mouse models, as well as joint delivery of TGF $\alpha-N P s$, demonstrate that it is feasible to precisely control EGFR signaling for cartilage anabolic actions selectively without incurring undesired catabolic effects. These observations also suggest that future study of TGF $\alpha$-NPs should optimize dosage, duration, and ligand specificity to achieve the best therapeutic effects.

Initial findings revealed that TGF $\alpha$ (30) and HBEGF (44) are elevated in degenerated OA cartilage. Accordingly, it was proposed that EGFR inhibitors could be repurposed for OA treatment. To date, the results of EGFR inhibitors on rodent OA progression are mixed and often contradictory, depending on the gender and type of inhibitors used in experiments (29). In our hands, we constantly observed accelerated OA by gefitinib treatment (25). Our previous study clearly showed that EGFR activity is most concentrated in the superficial layer of articular cartilage, which is drastically reduced during OA initiation (8). In this study, overactivation of EGFR in animal models, particularly using inducible Aggrecan-CreER or by TGF $\alpha-N P$ injections, demonstrated that elevating EGFR activation at the early stage of OA was beneficial. We also provided evidence that TGF $\alpha$-NP treatment starting from 1 month after DMM surgery remained effective. The underlying mechanisms, as shown here and in our previous studies, involve the protection of superficial layer from OA-induced destruction. At a later stage when the superficial zone is disrupted and TGF $\alpha$ and HBEGF are elevated, whether further increasing EGFR activity could still render a protective or even reparative effect on cartilage needs further investigation. During OA degeneration, new chondrocyte cell clusters are frequently formed under the damaged cartilage surface in an attempt to repair and regenerate (51). Those cell clusters are proliferative with stem cell properties. However, under normal circumstances, impaired cartilage does not repair by itself. We speculate that as growth factors for mesenchymal progenitors, TGF $\alpha$ and HBEGF are likely to be up-regulated for forming cell clusters after damage, yet their endogenous expression is not sufficiently high to regenerate the cartilage. Therefore, exogenous EGFR ligand might be helpful in attenuating OA progression even at a late stage.

One additional concern of our treatment approach is the possible carcinogenic effect of activating EGFR signaling. High EGFR activities due to missense mutations, deletions, and insertions are often observed in breast, lung, liver, brain tumors, etc. (52). In our study, we attempted to minimize this possibility by locally overactivating EGFR in cartilage. No obvious changes were observed in vital internal organs in mice overexpressing HBEGF specifically in cartilage up to 12 months of age or in mice intra-articularly injected with TGF $\alpha$-NPs. However, long-term pharmacological assays should be performed before translating our findings into the clinic. 
There is a great unmet medical need for a disease-modifying OA drug. In this study, we have demonstrated the feasibility of targeting EGFR signaling to block OA initiation and constructed a drug for such treatment without obvious side effects. This is just the initial step toward the translation of our findings into clinical usage. Because most patients with OA are already at middle or late OA stages, future experiments should test the efficacy of TGF $\alpha$-NPs after OA has well developed using large-animal models. Optimization of the structure of conjugates, likely using different EGFR ligands, and the dosage of conjugates should be explored to achieve favorable treatment outcomes. Such comprehensive studies will shed light on developing disease-modifying drugs that benefit patients with OA.

\section{MATERIALS AND METHODS Study design}

This study was performed to evaluate whether activating EGFR signaling specifically in joint cartilage could protect articular cartilage from OA degeneration. This objective was addressed by (i) examining articular cartilage phenotype in $H B E G F$ Over ${ }^{\text {Col2 }}$ and HBEGF Over ${ }^{\text {AgcER }}$ mice, (ii) delineating the cellular and molecular changes in their articular cartilage, (iii) characterizing their responses toward OA surgery, (iv) synthesizing and characterizing TGF $\alpha$-NPs, and (v) studying the effect of intra-articular injection of TGF $\alpha-N P s$ on attenuating OA progression. Sample size was determined by the investigators according to previous experimental experience. The exact $n$ numbers used in each experiment are indicated in the respective figure legends. For in vivo experiments, data from animals that died or had severe health problems during the experiments were excluded. Samples were assigned randomly to the experimental and control groups. Animal or sample allocation and data acquisition in vivo or ex vivo were performed in a blinded manner. The investigators were not blinded during data analysis.

\section{Animals}

All animal work performed in this study was approved by the Institutional Animal Care and Use Committee at the University of Pennsylvania. In accordance with the standards for animal housing, mice were group housed at $23^{\circ}$ to $25^{\circ} \mathrm{C}$ with a 12 -hour light/dark cycle and allowed free access to water and standard laboratory pellets.

Col2-Cre mice or Aggrecan-CreER mice were bred with Rosa-DTR mice to generate Col2-Cre DTR (HBEGF Over ${ }^{\mathrm{Col} 2}$ ) and Aggrecan-CreER DTR (HBEGF Over $\left.{ }^{A g c E R}\right)$ mice, respectively, and their WT (DTR- or Cre-only) siblings. All mouse lines were purchased from the Jackson laboratory.

To induce OA, male mice 3 months of age were subjected to DMM surgery or sham surgery at right knees as described previously (25). Briefly, in DMM surgery, the joint capsule was opened immediately after anesthesia, and the medial meniscotibial ligament was cut to destabilize the meniscus without damaging other tissues. In sham surgery, the joint capsule was opened in the same fashion but without any further damage.

HBEGF Over ${ }^{A g c E R}$ mice and WT controls received tamoxifen (SigmaAldrich) injections ( $75 \mathrm{mg} / \mathrm{kg}$ per day) for 5 days before DMM surgery at 3 months of age. For the EGFR inhibitor treatment study, these mice additionally received vehicle ( $0.5 \%$ methyl cellulose) or gefitinib (100 mg/kg; LC Laboratories) via oral gavage once every other day for 8 weeks after surgery. For EdU incorporation study, 3 -day-old mice received intraperitoneal injections of EdU $(2.5 \mathrm{mg} / \mathrm{kg}$;
Thermo Fisher Scientific) for 4 days. Knee joints were harvested on the fifth day or 3 weeks later.

For the TGF $\alpha$-NP treatment study, male $C 57 \mathrm{Bl} / 6$ mice (the Jackson laboratory) were randomly divided into five groups: sham surgery (sham), DMM and PBS treatment, DMM and free TGF $\alpha-\mathrm{DBCO}$ treatment (TGF $\alpha-\mathrm{DBCO}), \mathrm{DMM}$ and Ctrl-NP treatment (Ctrl-NP), and DMM and TGF $\alpha-N P$ treatment (TGF $\alpha-N P$ ). Treatments were given by intra-articular injection of $10 \mu \mathrm{l}$ of PBS, TGF $\alpha$-DBCO (10 $\mu \mathrm{M}$ TGF $\alpha$ content), Ctrl-NPs (NP with no TGF $\alpha$ conjugation), or TGF $\alpha$-NPs (10 $\mu \mathrm{M}$ TGF $\alpha$ content) once every 3 weeks initiated immediately after DMM surgery. Mice were euthanized 2 months after surgery (receiving a total number of three injections) or 3 months after surgery (receiving four injections).

\section{TGF $\alpha-N P$ synthesis}

TGF $\alpha$-NPs were prepared via click reaction (27). Briefly, stock solutions of poly(ethylene glycol) (4000)-polycaprolactone (3000) copolymer (denoted as PEG-PCL; Polymer Source), polylysine (3300)-polycaprolactone (4000) copolymer (denoted PLL-PCL), and 1,2-distearoyl-sn-glycero-3-phosphoethanolamine- $N$-[azido(polyethylene glycol)-5000] (ammonium salt) (denoted as DSPE-PEG5K-N ${ }_{3}$; Avanti Polar Lipids Inc) in chloroform (Sigma-Aldrich) were mixed in the following molar ratios: PEG-PCL/PLL-PCL/DSPE-PEG5K-N 3 $(55 / 20 / 25)$. The total amount of PEG-PCL for each of the NP compositions was $1 \mathrm{mg}$. The chloroform was removed using a direct stream of nitrogen before vacuum desiccation for overnight. NPs were formed by adding an aqueous solution (0.1 M PBS, pH 7.4) to the dried film and incubating in a $60^{\circ} \mathrm{C}$ water bath for 3 min and then sonicating for another $3 \mathrm{~min}$ at the same temperature. To prepare TGF $\alpha$-NPs, azide-modified NPs were mixed with TGF $\alpha$-DBCO at a molar ratio of 1 to 1 in $0.1 \mathrm{M} \mathrm{PBS}(\mathrm{pH} 7.4)$. Reactions were mixed overnight at room temperature and then purified by centrifugal filter devices (Amicon Ultra-4, 50K MWCO, Millipore Corp.). Samples were stored in the dark at $4^{\circ} \mathrm{C}$.

\section{Cartilage explant, cell culture, immunoblotting, and real-time reverse transcription PCR analysis}

Chondroprogenitors were harvested from articular cartilage of 5-month-old mouse knee joints. Briefly, cartilage was peeled from femoral condyles and tibial plateau by sterile scalpel under dissection microscope and incubated in $0.25 \%$ trypsin (Invitrogen) for 1 hour, followed by 2 hours of digestion with type I collagenase (900 U/ml; Worthington Biochemical). Dissociated cells from the second digestion were cultured in Gibco Dulbecco's modified Eagle's medium (DMEM) medium containing 10\% fetal bovine serum (FBS), streptomycin $(100 \mu \mathrm{g} / \mathrm{ml})$, and penicillin $(100 \mathrm{U} / \mathrm{ml})$. For CFU-F assay, $1 \times 10^{4}$ cells were seeded in a six-well plate and cultured for 7 days followed by crystal violet staining. CFU number was counted under microscope. For proliferation assay, cells were seeded in culture medium. Cell counting was performed on the indicated days. For apoptosis assay, cells at 40 to $60 \%$ confluency were serum starved overnight and then pretreated with either vehicle or TNF $\alpha$ (25 ng/ml; Pepro-Tech). Two days later, apoptotic cells were quantified using ethidium bromide ( $5 \mathrm{mg} / \mathrm{ml}$; Sigma-Aldrich) and acridine orange $(5 \mathrm{mg} / \mathrm{ml}$; Thermo Fisher Scientific) staining as described previously (53). For chondrogenic differentiation assay, confluent cells were cultured in differentiation medium [DMEM, streptomycin $(100 \mu \mathrm{g} / \mathrm{ml})$, penicillin $(100 \mathrm{U} / \mathrm{ml}), 1 \%$ insulin-transferrinselenous acid (ITS)+ Premix (Corning), L-proline $(40 \mu \mathrm{g} / \mathrm{ml}), 0.1 \mu \mathrm{M}$ 
dexamethasone, sodium pyruvate $(100 \mu \mathrm{g} / \mathrm{ml})$, ascorbate 2-phosphate $(50 \mu \mathrm{g} / \mathrm{ml})$, and TGF $33(10 \mathrm{ng} / \mathrm{ml})]$. Media were changed twice a week.

Synovial mesenchymal progenitors were harvested from 5 -monthold mouse knee joints as described previously with minor modifications (54). Briefly, the quadricep reversing approach was used to harvest mouse synovium, which was then incubated in $0.25 \%$ trypsin for 0.5 hours, followed by 1-hour digestion with type I collagenase $(300 \mathrm{U} / \mathrm{ml})$. Dissociated cells from the second digestion were cultured in DMEM medium containing 10\% FBS, streptomycin $(100 \mu \mathrm{g} / \mathrm{ml})$, and penicillin $(100 \mathrm{U} / \mathrm{ml})$. For CFU-F assay, $4 \times 10^{3}$ cells were seeded in a six-well plate and cultured for 10 days followed by crystal violet staining. CFU-F number was counted under microscope.

Human articular cartilage was prepared from the deidentified specimens obtained at the total arthroplasty of the knee joints. Explants were harvested from femoral condyle area with normal cartilage morphology using biopsy punch $(3 \mathrm{~mm}$ in diameter and $2 \mathrm{~mm}$ in thickness) and cultured in chemically defined medium [DMEM, streptomycin $(100 \mu \mathrm{g} / \mathrm{ml})$, penicillin $(100 \mathrm{U} / \mathrm{ml}), 1 \%$ ITS+ Premix, L-proline $(40 \mu \mathrm{g} / \mathrm{ml}), 0.1 \mu \mathrm{M}$ dexamethasone, sodium pyruvate $(100 \mu \mathrm{g} / \mathrm{ml})$, and ascorbate 2 -phosphate $(50 \mu \mathrm{g} / \mathrm{ml})]$ in a 48 -well plate. TGF $\alpha$-NPs were added to the medium with a final TGF $\alpha$ concentration of $100 \mathrm{ng} / \mathrm{ml}$. After 4 days of incubation with a medium change on day 3, cartilage explants were washed three times with PBS and processed either for RNA extraction or for paraffin sectioning.

To perform Western blot, cell lysate was solubilized in radioimmunoprecipitation assay buffer [50 mM tris $(\mathrm{pH} 7.4), 100 \mathrm{mM}$ $\mathrm{NaCl}, 1 \%$ sodium deoxycholate, $1 \%$ Triton X-100, and $0.1 \%$ SDS] with protease inhibitor (Sigma-Aldrich). Cell lysate $(50 \mathrm{mg}$ ) was separated by SDS-polyacrylamide gel electrophoresis (Bio-Rad) and transferred onto polyvinylidene difluoride (Bio-Rad) membrane. Immunoreactive protein bands were visualized using rabbit primary antibodies against EGFR (1:1000; CST, 4267), p-EGFR (1:1000; Abcam, ab40815), ERK (1:1000; CST, 4695), p-ERK (1:1000; CST, 4370), HBEGF/DTR (1:1000; Novus Biologicals, AF8239), $\beta$-Actin (1:3000; CST, 4970), and secondary antibodies (Vector Laboratories), followed by chemiluminescence (Amersham ECL Western Blotting Detection Reagents, GE Healthcare).

RNA was harvested from chondrocyte culture using Tri Reagent (Sigma-Aldrich). TaqMan Reverse Transcription kit (Applied Biosystems) was used to reverse transcribe mRNA into complementary DNA. After this, polymerase chain reaction (PCR) was performed using a Power SYBR Green PCR Master Mix kit (Applied Biosystems). The primer sequences for the genes used in this study are listed in table S1.

\section{Statistical analysis}

Data are expressed as means \pm SEM and analyzed by $t$ tests, oneway analysis of variance (ANOVA) with Dunnett's or Tukey's posttest, and two-way ANOVA with Bonferroni's or Tukey's posttest for multiple comparisons using Prism 8 software (GraphPad Software). For cell culture experiments, observations were repeated independently at least three times with a similar conclusion, and only data from a representative experiment are presented. Values of $P<0.05$ were considered significant.

\section{SUPPLEMENTARY MATERIALS}

stm.sciencemag.org/cgi/content/full/13/576/eabb3946/DC1 Materials and Methods

Fig. S1. HBEGF Over $\mathrm{Col}^{2}$ mice have normal body weight and body length.
Fig. S2. HBEGF Over ${ }^{\mathrm{Col} 2}$ mice overexpress HBEGF in cartilage.

Fig. S3. HBEGF Over ${ }^{\mathrm{Col} 2}$ mice have normal joint structure.

Fig. S4. Overexpressing HBEGF in articular cartilage does not affect long bone structure. Fig. S5. Mesenchymal progenitors in synovium are not affected by HBEGF overexpression in cartilage.

Fig. S6. Overexpressing HBEGF in cartilage does not affect cartilage matrix composition and cartilage degradation.

Fig. S7. Overexpressing HBEGF in cartilage does not affect vital internal organs.

Fig. S8. HBEGF Over ${ }^{A g c E R}$ mice have increased HBEGF expression and EGFR activity in knee articular cartilage.

Fig. S9. Synthesis and characterization of PLL-PCL.

Fig. S10. TGF $\alpha$-NPs result in similar morphology changes in chondrocytes as free TGF $\alpha$. Fig. S11. TGF $\alpha$-NPs are functional on human articular cartilage.

Fig. S12. TGF $\alpha$-NPs doped with PLL-PCL enhance bovine cartilage uptake.

Fig. S13. TGF $\alpha$-NPs doped with PLL-PCL improve their penetration and retention in bovine cartilage tissue.

Fig. S14. Biodistribution of TGF $\alpha$-NPs within the knee joints and some major organs. Fig. S15. TGF $\alpha$-NP treatment attenuates OA progression after DMM surgery in mice. Fig. S16. TGF $\alpha$-NP treatment starting at 1 month after DMM attenuates further OA progression. Fig. S17. The mechanism underlying the protective action of EGFR overactivation on articular cartilage after DMM surgery.

Fig. S18. Intra-articular injections of TGF $\alpha$-NPs for 2 months do not affect vital internal organs and gross joint morphology.

Table S1. Mouse real-time PCR primer sequences.

Data file S1. Individual subject-level data (Excel file).

Data file S2. Western blotting films (PDF).

References (55-59)

View/request a protocol for this paper from Bio-protocol.

\section{REFERENCES AND NOTES}

1. GBD 2017 Disease and Injury Incidence and Prevalence Collaborators, Global, regional, and national incidence, prevalence, and years lived with disability for 354 diseases and injuries for 195 countries and territories, 1990-2017: A systematic analysis for the Global Burden of Disease Study 2017. Lancet 392, 1789-1858 (2018).

2. R. F. Loeser, S. R. Goldring, C. R. Scanzello, M. B. Goldring, Osteoarthritis: A disease of the joint as an organ. Arthritis Rheumatol. 64, 1697-1707 (2012).

3. J. Becerra, J. A. Andrades, E. Guerado, P. Zamora-Navas, J. M. Lopez-Puertas, A. H. Reddi, Articular cartilage: Structure and regeneration. Tissue Eng. Part B Rev. 16, 617-627 (2010).

4. S. S. Glasson, M. G. Chambers, W. B. Van Den Berg, C. B. Little, The OARSI histopathology initiative-Recommendations for histological assessments of osteoarthritis in the mouse. Osteoarthr. Cartil. 18, S17-S23 (2010).

5. K. P. Pritzker, S. Gay, S. A. Jimenez, K. Ostergaard, J. P. Pelletier, P. A. Revell, D. Salter, W. B. van den Berg, Osteoarthritis cartilage histopathology: Grading and staging. Osteoarthr. Cartil. 14, 13-29 (2006).

6. B. Rolauffs, M. Rothdiener, C. Bahrs, A. Badke, K. Weise, K. E. Kuettner, B. Kurz, M. Aurich, A. J. Grodzinsky, W. K. Aicher, Onset of preclinical osteoarthritis: The angular spatial organization permits early diagnosis. Arthritis Rheumatol. 63, 1637-1647 (2011).

7. A. Citri, Y. Yarden, EGF-ERBB signalling: Towards the systems level. Nat. Rev. Mol. Cell Biol. 7, 505-516 (2006).

8. H. Jia, X. Ma, W. Tong, B. Doyran, Z. Sun, L. Wang, X. Zhang, Y. Zhou, F. Badar, A. Chandra, X. L. Lu, Y. Xia, L. Han, M. Enomoto-Iwamoto, L. Qin, EGFR signaling is critical for maintaining the superficial layer of articular cartilage and preventing osteoarthritis initiation. Proc. Natl. Acad. Sci. U.S.A. 113, 14360-14365 (2016).

9. H. Jia, X. Ma, Y. Wei, W. Tong, R. J. Tower, A. Chandra, L. Wang, Z. Sun, Z. Yang, F. Badar, K. Zhang, W. J. Tseng, I. Kramer, M. Kneissel, Y. Xia, X. S. Liu, J. H. Wang, L. Han, M. Enomoto-Iwamoto, L. Qin, Loading-induced reduction in Sclerostin as a mechanism of subchondral bone plate sclerosis in mouse knee joints during late-stage osteoarthritis. Arthritis Rheumatol. 70, 230-241 (2018).

10. X. Zhang, K. A. Pickin, R. Bose, N. Jura, P. A. Cole, J. Kuriyan, Inhibition of the EGF receptor by binding of MIG6 to an activating kinase domain interface. Nature 450, 741-744 (2007).

11. M. A. Pest, B. A. Russell, Y. W. Zhang, J. W. Jeong, F. Beier, Disturbed cartilage and joint homeostasis resulting from a loss of mitogen-inducible gene 6 in a mouse model of joint dysfunction. Arthritis Rheumatol. 66, 2816-2827 (2014).

12. J. B. Shepard, J. W. Jeong, N. J. Maihle, S. O'Brien, C. N. Dealy, Transient anabolic effects accompany epidermal growth factor receptor signal activation in articular cartilage in vivo. Art Ther. 15, R60 (2013).

13. B. Staal, B. O. Williams, F. Beier, G. F. Vande Woude, Y. W. Zhang, Cartilage-specific deletion of Mig-6 results in osteoarthritis-like disorder with excessive articular chondrocyte proliferation. Proc. Natl. Acad. Sci. U.S.A. 111, 2590-2595 (2014). 
14. T. Buch, F. L. Heppner, C. Tertilt, T. J. A. J. Heinen, M. Kremer, F. T. Wunderlich, S. Jung, A. Waisman, A Cre-inducible diphtheria toxin receptor mediates cell lineage ablation after toxin administration. Nat. Methods 2, 419-426 (2005).

15. R. Iwamoto, S. Higashiyama, T. Mitamura, N. Taniguchi, M. Klagsbrun, E. Mekada, Heparin-binding EGF-like growth factor, which acts as the diphtheria toxin receptor, forms a complex with membrane protein DRAP27/CD9, which up-regulates functional receptors and diphtheria toxin sensitivity. EMBO J. 13, 2322-2330 (1994).

16. W. Pan, K. Vallance, A. J. Kastin, TGF $\alpha$ and the blood-brain barrier: Accumulation in cerebral vasculature. Exp. Neurol. 160, 454-459 (1999).

17. B. C. Geiger, S. Wang, R. F. Padera Jr., A. J. Grodzinsky, P. T. Hammond, Cartilagepenetrating nanocarriers improve delivery and efficacy of growth factor treatment of osteoarthritis. Sci. Transl. Med. 10, eaat8800 (2018).

18. S. Kumar, I. M. Adjei, S. B. Brown, O. Liseth, B. Sharma, Manganese dioxide nanoparticles protect cartilage from inflammation-induced oxidative stress. Biomaterials 224, 119467 (2019).

19. H. Yan, X. Duan, H. Pan, N. Holguin, M. F. Rai, A. Akk, L. E. Springer, S. A. Wickline, L. J. Sandell, C. T. Pham, Suppression of NF- $\mathrm{KB}$ activity via nanoparticle-based siRNA delivery alters early cartilage responses to injury. Proc. Natl. Acad. Sci. U.S.A. 113, E6199-E6208 (2016).

20. E. Kozhemyakina, M. Zhang, A. lonescu, U. M. Ayturk, N. Ono, A. Kobayashi, H. Kronenberg, M. L. Warman, A. B. Lassar, Identification of a Prg4-expressing articular cartilage progenitor cell population in mice. Arthritis Rheumatol. 67, 1261-1273 (2015).

21. U. Sahin, G. Weskamp, K. Kelly, H. M. Zhou, S. Higashiyama, J. Peschon, D. Hartmann, P. Saftig, C. P. Blobel, Distinct roles for ADAM10 and ADAM17 in ectodomain shedding of six EGFR ligands. J. Cell Biol. 164, 769-779 (2004).

22. B. Doyran, W. Tong, Q. Li, H. Jia, X. Zhang, C. Chen, M. Enomoto-Iwamoto, X. L. Lu, L. Qin, L. Han, Nanoindentation modulus of murine cartilage: A sensitive indicator of the initiation and progression of post-traumatic osteoarthritis. Osteoarthr. Cartil. 25, 108-117 (2017).

23. K. Elenius, S. Paul, G. Allison, J. Sun, M. Klagsbrun, Activation of HER4 by heparin-binding EGF-like growth factor stimulates chemotaxis but not proliferation. EMBO J. 16, 1268-1278 (1997).

24. C. Yewale, D. Baradia, I. Vhora, S. Patil, A. Misra, Epidermal growth factor receptor targeting in cancer: A review of trends and strategies. Biomaterials 34, 8690-8707 (2013).

25. X. Zhang, J. Zhu, F. Liu, Y. Li, A. Chandra, L. S. Levin, F. Beier, M. Enomoto-Iwamoto, L. Qin Reduced EGFR signaling enhances cartilage destruction in a mouse osteoarthritis model. Bone Res. 2, 14015 (2014).

26. B. Singh, R. J. Coffey, From wavy hair to naked proteins: The role of transforming growth factor alpha in health and disease. Semin. Cell Dev. Biol. 28, 12-21 (2014).

27. J. Z. Hui, A. Al Zaki, Z. Cheng, V. Popik, H. Zhang, E. T. Luning Prak, A. Tsourkas, Facile method for the site-specific, covalent attachment of full-length IgG onto nanoparticles. Small 10, 3354-3363 (2014)

28. R. Warden-Rothman, I. Caturegli, V. Popik, A. Tsourkas, Sortase-tag expressed protein ligation: Combining protein purification and site-specific bioconjugation into a single step. Anal. Chem. 85, 11090-11097 (2013).

29. L. Qin, F. Beier, EGFR signaling: Friend or foe for cartilage? JBMR Plu 3, e10177 (2019).

30. C. T. Appleton, S. E. Usmani, S. M. Bernier, T. Aigner, F. Beier, Transforming growth factor alpha suppression of articular chondrocyte phenotype and Sox 9 expression in a rat model of osteoarthritis. Arthritis Rheumatol. 56, 3693-3705 (2007).

31. A. J. Sophia Fox, A. Bedi, S. A. Rodeo, The basic science of articular cartilage: Structure, composition, and function. Sports Health 1, 461-468 (2009).

32. A. Mathiessen, P. G. Conaghan, Synovitis in osteoarthritis: Current understanding with therapeutic implications. Arthritis Res. Ther. 19, 18 (2017).

33. J. Marcelino, J. D. Carpten, W. M. Suwairi, O. M. Gutierrez, S. Schwartz, C. Robbins, R. Sood, I. Makalowska, A. Baxevanis, B. Johnstone, R. M. Laxer, L. Zemel, C. A. Kim, J. K. Herd, J. Ihle, C. Williams, M. Johnson, V. Raman, L. G. Alonso, D. Brunoni, A. Gerstein, N. Papadopoulos, S. A. Bahabri, J. M. Trent, M. L. Warman, CACP, encoding a secreted proteoglycan, is mutated in camptodactyly-arthropathy-coxa vara-pericarditis syndrome. Nat. Genet. 23, 319-322 (1999).

34. J. M. Coles, L. Zhang, J. J. Blum, M. L. Warman, G. D. Jay, F. Guilak, S. Zauscher, Loss of cartilage structure, stiffness, and frictional properties in mice lacking PRG4. Arthritis Rheumatol. 62, 1666-1674 (2010).

35. C. R. Flannery, R. Zollner, C. Corcoran, A. R. Jones, A. Root, M. A. Rivera-Bermúdez, T. Blanchet, J. P. Gleghorn, L. J. Bonassar, A. M. Bendele, E. A. Morris, S. S. Glasson, Prevention of cartilage degeneration in a rat model of osteoarthritis by intraarticular treatment with recombinant lubricin. Arthritis Rheumatol. 60, 840-847 (2009).

36. E. R. Sampson, M. J. Hilton, Y. Tian, D. Chen, E. M. Schwarz, R. A. Mooney, S. V. Bukata, R. J. O'Keefe, H. Awad, J. E. Puzas, R. N. Rosier, M. J.Zuscik, Teriparatide as a chondroregenerative therapy for injury-induced osteoarthritis. Sci. Transl. Med. 3, 101 ra193 (2011).

37. T. Uchimura, A. T. Foote, E. L. Smith, E. G. Matzkin, L. Zeng, Insulin-like growth factor II (IGF-II) inhibits IL-1 $\beta$-induced cartilage matrix loss and promotes cartilage integrity in experimental osteoarthritis. J. Cell. Biochem. 116, 2858-2869 (2015).

38. E. E. Moore, A. M. Bendele, D. L. Thompson, A. Littau, K. S. Waggie, B. Reardon, J. L. Ellsworth, Fibroblast growth factor- 18 stimulates chondrogenesis and cartilage repair in a rat model of injury-induced osteoarthritis. Osteoarthr. Cartil. 13, 623-631 (2005).
39. Y. I. Jeong, J. B. Cheon, S. H. Kim, J. W. Nah, Y. M. Lee, Y. K. Sung, T. Akaike, C. S. Cho, Clonazepam release from core-shell type nanoparticles in vitro. J. Control. Release 51, 169-178 (1998).

40. A. Al Zaki, D. Joh, Z. L. Cheng, A. L. B. De Barros, G. Kao, J. Dorsey, A. Tsourkas, Gold-loaded polymeric micelles for computed tomography-guided radiation therapy treatment and radiosensitization. ACS Nano 8, 104-112 (2013).

41. A. V. Kabanov, E. V. Batrakova, N. S. Meliknubarov, N. A. Fedoseev, T. Y. Dorodnich, V. Y. Alakhov, V. P. Chekhonin, I. R. Nazarova, V. A. Kabanov, A new class of drug carriersMicelles of poly(oxyethylene)-poly(oxypropylene) block copolymers as microcontainers for drug targeting from blood in brain. J. Control. Release 22, 141-157 (1992).

42. H. Cabral, K. Miyata, K. Osada, K. Kataoka, Block copolymer micelles in nanomedicine applications. Chem. Rev. 118, 6844-6892 (2018).

43. X. Zhang, J. Zhu, Y. Li, T. Lin, V. A. Siclari, A. Chandra, E. M. Candela, E. Koyama, M. Enomoto-Iwamoto, L. Qin, Epidermal growth factor receptor (EGFR) signaling regulates epiphyseal cartilage development through $\beta$-catenin-dependent and-independent pathways. J. Biol. Chem. 288, 32229-32240 (2013).

44. D. L. Long, V. Ulici, S. Chubinskaya, R. F. Loeser, Heparin-binding epidermal growth factor-like growth factor (HB-EGF) is increased in osteoarthritis and regulates chondrocyte catabolic and anabolic activities. Osteoarthr. Cartil. 23, 1523-1531 (2015).

45. Y. W. Zhang, Y. Su, N. Lanning, P. J. Swiatek, R. T. Bronson, R. Sigler, R. W. Martin, G. F. Vande Woude, Targeted disruption of Mig- 6 in the mouse genome leads to early onset degenerative joint disease. Proc. Natl. Acad. Sci. U.S.A. 102, 11740-11745 (2005).

46. P. Li, Q. Deng, J. Liu, J. Yan, Z. Wei, Z. Zhang, H. Liu, B. Li, Roles for HB-EGF in mesenchymal stromal cell proliferation and differentiation during skeletal growth. J. Bone Miner. Res. 34, 295-309 (2019).

47. K. Yu, J. Xu, Z. Liu, D. Sosic, J. Shao, E. N. Olson, D. A. Towler, D. M. Ornitz, Conditional inactivation of FGF receptor 2 reveals an essential role for FGF signaling in the regulation of osteoblast function and bone growth. Development 130, 3063-3074 (2003).

48. D. A. Ovchinnikov, J. M. Deng, G. Ogunrinu, R. R. Behringer, Col2a1-directed expression of Cre recombinase in differentiating chondrocytes in transgenic mice. Genesis 26, $145-146$ (2000).

49. S. P. Henry, C. W. Jang, J. M. Deng, Z. Zhang, R. R. Behringer, B. de Crombrugghe, Generation of aggrecan-CreERT2 knockin mice for inducible Cre activity in adult cartilage. Genesis 47, 805-814 (2009).

50. G. Pante, J. Thompson, F. Lamballe, T. Iwata, I. Ferby, F. A. Barr, A. M. Davies, F. Maina, R. Klein, Mitogen-inducible gene 6 is an endogenous inhibitor of HGF/Met-induced cell migration and neurite growth. J. Cell Biol. 171, 337-348 (2005).

51. M. K. Lotz, S. Otsuki, S. P. Grogan, R. Sah, R. Terkeltaub, D. D'Lima, Cartilage cell clusters. Arthritis Rheumatol. 62, 2206-2218 (2010).

52. R. Roskoski Jr., The ErbB/HER family of protein-tyrosine kinases and cancer. Pharmacol. Res. 79, 34-74 (2014).

53. A. Chandra, S. Lan, J. Zhu, V. Siclari, L. Qin, Epidermal growth factor receptor (EGFR) signaling promotes proliferation and survival in osteoprogenitors by increasing early growth response 2 (Egr2) expression. J. Biol. Chem. 288, 20488-20498 (2013).

54. I. Futami, M. Ishijima, H. Kaneko, K. Tsuji, N. Ichikawa-Tomikawa, R. Sadatsuki, T. Muneta, E. Arikawa-Hirasawa, I. Sekiya, K. Kaneko, Isolation and characterization of multipotential mesenchymal cells from the mouse synovium. PLOS ONE 7, e45517 (2012).

55. C. D. DiDomenico, Z. Xiang Wang, L. J. Bonassar, Cyclic mechanical loading enhances transport of antibodies into articular cartilage. J. Biomech. Eng. 139, doi:10.1115/1.4035265 (2017).

56. V. Krenn, L. Morawietz, T. Haupl, J. Neidel, I. Petersen, A. Konig, Grading of chronic synovitis-A histopathological grading system for molecular and diagnostic pathology. Pathol. Res. Pract. 198, 317-325 (2002).

57. T. Aigner, J. L. Cook, N. Gerwin, S. S. Glasson, S. Laverty, C. B. Little, W. Mcllwraith, V. B. Kraus, Histopathology atlas of animal model systems-Overview of guiding principles. Osteoarthr. Cartil. 18 (Suppl 3), S2-S6 (2010).

58. M. A. Batista, H. T. Nia, P. Onnerfjord, K. A. Cox, C. Ortiz, A. J. Grodzinsky, D. Heinegard, L. Han, Nanomechanical phenotype of chondroadherin-null murine articular cartilage. Matrix Biol. 38, 84-90 (2014).

59. M. J. Piel, J. S. Kroin, H. J. Im, Assessment of knee joint pain in experimental rodent models of osteoarthritis. Methods Mol. Biol. 1226, 175-181 (2015).

\section{Acknowledgments}

Funding: This study was supported by NIH grants R01AR066098, R01DK095803, R01AG067698 (to L.Q.), P30AR069619 (to the Penn Center for Musculoskeletal Disorders), AR074490 (to L.H.), and R01NS100892 (to Z.C.). Author contributions: L.Q. and Z.C. designed the study. Y.W., L.L., L.Yao, and T.G. performed animal experiments. L.L., F.Y., and L.Yan made NP formulations. Y.W., L.L., L.Yao, W.Y., L.Z., and T.G. performed histology and imaging analysis. Y.W., J.F.L., L.Yao, and T.G. performed cell culture and qRT-PCR experiments. B.H. and L.H. performed the nanoindentation experiments. J.F.L. calculated the diffusivity. J.M.P., F.B., Z.S., L.H., R.L.M., A.T., 
J.A., L.S.L., and C.N. provided administrative, technical, or material support and consultation. L.Q. and Z.C. wrote the manuscript. F.B., Z.S., L.H., R.L.M., A.T., and J.A. reviewed and revised the manuscript. L.Q. and Z.C. approved the final version. Competing interests: L.Q., Z.C., and Y.W. are listed on a patent associated with this manuscript, "Targeting Cartilage EGFR Pathway for Osteoarthritis Treatment," U.S. Provisional Patent Application no. 63/067,546. A.T. is an inventor on patent US20160032346A1, "Sortase-mediated protein purification and ligation," which was used for the site-specific modification of TGF $\alpha$, with DBCO. Data and materials availability: All data associated with this study are present in the paper or the Supplementary Materials.
Submitted 20 February 2020

Accepted 23 November 2020

Published 13 January 2021

10.1126/scitransImed.abb3946

Citation: Y. Wei, L. Luo, T. Gui, F. Yu, L. Yan, L. Yao, L. Zhong, W. Yu, B. Han, J. M. Patel, J. F. Liu, F. Beier, L. S. Levin, C. Nelson, Z. Shao, L. Han, R. L. Mauck, A. Tsourkas, J. Ahn, Z. Cheng, L. Qin Targeting cartilage EGFR pathway for osteoarthritis treatment. Sci. Transl. Med. 13, eabb3946 (2021) 


\title{
Science Translational Medicine
}

\section{Targeting cartilage EGFR pathway for osteoarthritis treatment}

Yulong Wei, Lijun Luo, Tao Gui, Feifan Yu, Lesan Yan, Lutian Yao, Leilei Zhong, Wei Yu, Biao Han, Jay M. Patel, Jessica F. Liu, Frank Beier, Lawrence Scott Levin, Charles Nelson, Zengwu Shao, Lin Han, Robert L. Mauck, Andrew Tsourkas, Jaimo Ahn, Zhiliang Cheng and Ling Qin

Sci Transl Med 13, eabb3946.

DOI: 10.1126/scitranslmed.abb3946

\begin{abstract}
Enhancing EGFR to offset osteoarthritis
Cartilage degeneration in osteoarthritis causes pain and limits mobility. Wei et al. investigated epidermal growth factor receptor (EGFR) signaling as a potential targeted disease-modifying treatment, because EGFR activity is linked to cartilage homeostasis. Cartilage-specific genetic overactivation of EGFR resulted in enlarged cartilage, an expanded tissue progenitor cell population, and resistance to surgically induced osteoarthritis in mice. Treating mice with transforming growth factor $-\alpha$ (an EGFR ligand) conjugated to nanoparticles via intra-articular injections protected against cartilage degeneration and attenuated pain in an osteoarthritis model. Results show how targeting EGFR signaling could potentially treat osteoarthritis.
\end{abstract}

ARTICLE TOOLS

http://stm.sciencemag.org/content/13/576/eabb3946

SUPPLEMENTARY MATERIALS

http://stm.sciencemag.org/content/suppl/2021/01/11/13.576.eabb3946.DC1

RELATED
CONTENT

http://stm.sciencemag.org/content/scitransmed/10/469/eaat8800.full http://stm.sciencemag.org/content/scitransmed/11/491/eaau8587.full http://stm.sciencemag.org/content/scitransmed/10/427/eaan5372.full http://stm.sciencemag.org/content/scitransmed/12/533/eaay1041.full http://stm.sciencemag.org/content/scitransmed/13/580/eaau8491.full

REFERENCES

This article cites 58 articles, 11 of which you can access for free http://stm.sciencemag.org/content/13/576/eabb3946\#BIBL

PERMISSIONS

http://www.sciencemag.org/help/reprints-and-permissions

Use of this article is subject to the Terms of Service

Science Translational Medicine (ISSN 1946-6242) is published by the American Association for the Advancement of Science, 1200 New York Avenue NW, Washington, DC 20005. The title Science Translational Medicine is a registered trademark of AAAS.

Copyright (C) 2021 The Authors, some rights reserved; exclusive licensee American Association for the Advancement of Science. No claim to original U.S. Government Works 